\title{
A triangular prism solid and shell interactive mapping element for electromagnetic sheet metal forming process
}

\author{
Xiangyang Cui ${ }^{\mathrm{a}, \mathrm{b},{ }^{*}}$, She $\mathrm{Li}^{\mathrm{a}}{ }^{\mathrm{b}}$, Hui Feng ${ }^{\mathrm{a}, \mathrm{b}}$, Guangyao $\mathrm{Li}^{\mathrm{a}, \mathrm{b}}$ \\ a State Key Laboratory of Advanced Design and Manufacturing for Vehicle Body, Hunan \\ University, Changsha 410082, PR China \\ ${ }^{\mathrm{b}}$ Collaborative Innovation Center of Intelligent New Energy Vehicle, Shanghai, 200092, PR \\ China
}

\begin{abstract}
In this paper, a novel triangular prism solid and shell interactive mapping element is proposed to solve the coupled magnetic-mechanical formulation in electromagnetic sheet metal forming process. A linear six-node "Triprism" element is firstly proposed for transient eddy current analysis in electromagnetic field. In present "Triprism" element, shape functions are given explicitly, and a cell-wise gradient smoothing operation is used to obtain the gradient matrices without evaluating derivatives of shape functions. In mechanical field analysis, a shear locking free triangular shell element is employed in internal force computation, and a data mapping method is developed to transfer the Lorentz force on solid into the external forces suffered by shell structure for dynamic elasto-plasticity deformation analysis. Based on the deformed triangular shell structure, a "Triprism" element generation rule is established for updated electromagnetic analysis, which means inter-transformation of meshes between the coupled fields can be performed automatically. In addition, the dynamic moving mesh is adopted for air mesh updating based on the deformation of sheet metal. A benchmark problem is carried out for confirming the accuracy of the proposed "Triprism" element in predicting flux density in electromagnetic field. Solutions of several EMF problems obtained by present work are compared with experiment results and those of traditional method, which are showing excellent performances of present interactive mapping element.
\end{abstract}

Key words: Electromagnetic sheet metal forming; Coupled magnetic-mechanical analysis; "Triprism" element; Shell element; Stable nodal integration method

\footnotetext{
* Corresponding author. Tel: 86-731-88821717; Fax: 86-731-88822051

Email address: cuixy@hnu.edu.cn
} 


\section{Introduction}

Electromagnetic sheet metal forming (EMF) is one of the high-speed forming methods. During the electromagnetic sheet metal forming process, eddy currents on sheet metal flow in the opposite direction when sheet is placed on the intense transient magnetic field created by a discharge current running through a coil. As a consequence, a large magnitude magnetic repulsion force is created between the coil and the sheet which is large enough to overcome the yield strength of the sheet and cause a permanent deformation. EMF process attracts increasing scientific interests owing to the significant properties in improving the formability of metal sheets and reducing spring-back and wrinkling [1,2]. The application prospects of EMF are excellent in solving the problem of forming lightweight metals that are difficult to shape.

The EMF process is a complicated multi-physics-coupled problem, such as electromagnetic $[3,4]$, mechanical $[5,6]$, and thermal $[7,8]$, that can hardly solve it with theoretical analysis. Fortunately, numerical simulation technique offers an opportunity to better understand it $[9,10]$. It is necessary to build a efficient modeling of EMF process for simulating and then to better guide the practical application. In the past, researchers simplified the EMF process as two specific problems (electromagnetic and mechanical problems), and 2D axisymmetric modeling was mainly employed. Takatsu et al. [11] proposed a coupled magnetic and mechanical field in metal bulging process. Fenton and Daehn [12] utilized a 2D arbitrary Lagrangian Eulerian (ALE) finite difference code to predict electromagnetic sheet metal bulging process, and the simulation result predicted the deformation. Recently, both loose coupling and sequential coupling are employed in EMF simulation. Mamalis et al. [13] used the loose coupling strategy for parameters estimation. Yu et al. [14] analyzed the tube compression problem with comparison of sequential coupling and loose coupling method, and found out that the improvement of accuracy by sequential coupling is limited in small deformation analysis. Cui et al. [15] pointed out that loose coupling method is more efficient when relative small deformation happened on the sheet metal. Bartels et al. [16] indicated that the deviations between the two strategies increase with time, and that the loose coupling approach can only be feasible for relatively fast deformation and small deformation process. Otherwise, sequential coupling method which considers the effect of the sheet metal deformation 
to electromagnetic field should be employed.

All above were simulated by 2D modeling, and Bahmani et al. [17] made a comparison of the 3D and 2D simulation results, which indicated that some higher errors would be resulted by $2 \mathrm{D}$ simulation. Moreover, the 3D modeling is crucial for an effective process design in industrial application. Therefore, 3D EMF modeling attracted many researchers, and many works were advanced in past years. Oliveira et al. [18] presented a loose coupling method based on ANSYS/EMAG and LS-DYNA for sheet free bulging analysis. Cui et al. [19] analyzed the tube expansion, and adopted the sequential coupled 3D simulation based on ANSYS/ MULTIPHYSICAL. To update the electromagnetic geometry based on the sheet metal deformation, Stiemer et al. [20] proposed an arbitrary Lagrangian Eulerian approach for 3D EMF process, Cui el al. [19] adopted a morphing method to update the air meshes, besides, "re-meshing" technique was also utilized commonly. However, in contrast to 2D modeling, the computational cost increases dramatically in the case of 3D EMF process modeling. The major reason of computationally expensive is that the time step size for stability is very small, which caused by very "thin" solid elements are employed in the sheet metal for structure dynamic large deformation analysis. To improve the efficiency, Unger et al. [21] adopted a solid-shell element proposed by Reese et al. [22] in mechanical field, the simulation time is reduced observably. Considering the geometry features of the sheet metal, triangular shell element, which needs much less computational cost, is an alternative in electromagnetic sheet metal forming simulation. Corresponding to triangular shell element, triangular prism solid is an appropriate one for electromagnetic analysis. In the past, researchers customarily defined the shape function of triangular prism element as hexahedral elements done. Flores [23, 24] proposed a triangular "Prism" solid element for large strain shell analysis, the mapping and co-ordinate transformation was still involved and the neighbor elements were employed to eliminate the locking phenomenon. However, large computation cost and few possibilities given by the interpolation functions of the standard "prism" element probably constrain the development. Recently, sub-domain gradient smoothing operation $[25,26]$, which is used to approximate the derivatives of the field function in global Galerkin weak forms, providing another alternative for these "prism" element.

In this paper, a linear six-node "Triprism" element with sub-domain gradient smoothing operation is firstly proposed for transient eddy current analysis in sheet 
metal, and a stable nodal integration method [27] based on tetrahedron elements is employed for electromagnetic analysis in complicated coil and air region without structure deformation. A shear locking free triangular shell element [28] is considered for structure dynamic large deformation analysis in electromagnetic sheet metal forming process. In present "Triprism" element, shape functions are given explicitly, and a cell-wise gradient smoothing operation is used to obtain the gradient matrices without evaluating derivatives of shape functions. In order to increase the accuracy and stability, each "Triprism" element is further subdivided into two smoothing cells over the thickness direction. Based on the "Triprism" solid element and triangular shell element, an interactive mapping is proposed for dealing with the data interaction between the electromagnetic and the mechanical field. In present interactive mapping, Lorentz forces obtained by "Triprism" elements are firstly gathered in the mid-surface as external forces for sheet metal deformation, and then the updated "Triprism" elements are generated based on the nodal coordinates of deformed triangular shell elements. In order to accurately predict the gradual spread of plasticity in shell, a layered approach is used in structure deformation analysis. Several electromagnetic forming examples demonstrate that the results of proposed modeling own enough accuracy and much less computational cost, which shows the potentialities of present work in further research and application.

\section{Formulations for electromagnetic field}

\subsection{Governing Equation}

The governing equations for electromagnetic transient problem in Cartesian coordinates can be written as

$$
\begin{gathered}
V: \nabla \times v \nabla \times \mathbf{A}=\mathbf{J}_{s} \\
\left\{\begin{array}{l}
V: \nabla \times(v \nabla \times \mathbf{A})+\sigma \frac{\partial \mathbf{A}}{\partial t}+\sigma \nabla \frac{\partial \varphi}{\partial t}=\mathbf{J}_{s} \\
V_{1}: \nabla \cdot\left(-\sigma \frac{\partial \mathbf{A}}{\partial t}-\sigma \nabla \frac{\partial \varphi}{\partial t}\right)=0
\end{array}\right.
\end{gathered}
$$

where $v$ is the magnetic reluctivity, $\sigma$ is the conductivity, $\mathbf{A}$ is the magnetic vector potential, $\varphi$ is the electric scalar potential, and $\mathbf{J}_{s}$ is the current density.

\subsection{Equation discretization}


Applying the shape function $N_{j}(j=1,2, \cdots, n)$, magnetic vector potential $\mathbf{A}$ and electric scalar potential $\varphi$ can be written as

$$
\hat{\mathbf{A}}=\sum_{j=1}^{n} N_{j} \mathbf{A}_{j}, \hat{\varphi}=\sum_{j=1}^{n} N_{j} \varphi_{j}
$$

where $\mathbf{A}_{j}, \varphi_{j}$ are the field variable value at the node and $N_{j}$ is the shape function of the finite element method.

The weak form of Eq. (1) can be obtained using the standard Galerkin method, which is expressed as

$$
\int_{\Omega} \mathbf{N}_{i} \cdot\left(\nabla \times v \nabla \times \mathbf{A}-\mathbf{J}_{s}\right) \mathrm{d} \Omega=\mathbf{0}
$$

Adopting the divergence theorem and applying boundary conditions, it obtains

$$
\int_{\Omega} \nabla \times \mathbf{N}_{i} \cdot(v \nabla \times \mathbf{A}) \mathrm{d} \Omega=\int_{\Omega} \mathbf{N}_{i} \cdot \mathbf{J}_{s} \mathrm{~d} \Omega
$$

Substituting Eq. (3) into Eq. (5), the discretized system equation can be given in the following matrix form

$$
\mathbf{K A}=\mathbf{F}
$$

In the same way, the weak form of Eq. (2) can be expressed as

$$
\begin{aligned}
& {\left[\begin{array}{cc}
\int_{V}\left[\nabla \times \mathbf{N}_{i} \cdot v \nabla \times()\right] d V & 0 \\
0 & 0
\end{array}\right] \cdot\left[\begin{array}{c}
\mathbf{A} \\
\varphi
\end{array}\right]+} \\
& {\left[\begin{array}{cc}
\int_{V} \sigma \mathbf{N}_{i} \cdot() d V & \int_{V} \sigma \mathbf{N}_{i} \cdot \nabla() d V \\
\int_{V_{1}} \sigma \nabla \mathbf{N}_{i} \cdot() d V & \int_{V_{1}} \sigma \nabla \mathbf{N}_{i} \cdot \nabla() d V
\end{array}\right]\left[\begin{array}{l}
\frac{\partial \mathbf{A}}{\partial t} \\
\frac{\partial \varphi}{\partial t}
\end{array}\right]} \\
& =\left[\begin{array}{c}
\int_{V} \mathbf{N}_{i} \mathbf{J}_{s} d V \\
0
\end{array}\right]
\end{aligned}
$$

and the matrix form is

$$
\mathbf{M} \frac{\partial \mathbf{A}}{\partial t}+\mathbf{K A}=\mathbf{F}
$$

in which $\mathbf{A}=\left\{\begin{array}{lll}A_{x} & A_{y} & A_{z}\end{array}\right\}^{\mathrm{T}}$, and all items of Eq. (8), such as $\mathbf{M}, \mathbf{A}$, and $\mathbf{F}$, can be expressed as integral equations of $N_{i}, N_{j}, \partial N_{i} / \partial r, \partial N_{j} / \partial r(r=x, y, z)$.

\subsection{Element formulation for electromagnetic analysis}

In present work, a six-node "Triprism" element is developed for transient eddy current computation, which provide convenience of mesh mapping and Lorentz 
force data interaction between the solid and shell structure. In coil and air region without structure deformation, a stable nodal integration method (SNIM) based on tetrahedron elements is adopted for electromagnetic analysis.

\subsection{1. "Triprism" element}

As shown in Fig. 1, the sheet metal domain $\Omega$ is firstly discretized into several "Triprism" elements. Sub-domain gradient smoothing operation $[29,30]$ is used to guarantee the accuracy and stability, which is the same as the idea of smoothed finite element method (SFEM) [31, 32]. In present "Triprism" element, each element is further subdivided into two non-overlapping cells $\Omega_{s c 1}$ and $\Omega_{s c 2}$ and the values of the shape function are indicated explicitly at the nodes $(1,2,3,4,5,6)$. Then, the shape function values of other points are obtained by linear interpolation of those at given nodes.

In electromagnetic field, magnetic flux density $\mathbf{B}$ is defined as

$$
\mathbf{B}=\nabla \times \mathbf{A}=\left(\frac{\partial A_{z}}{\partial y}-\frac{\partial A_{y}}{\partial z}\right) \cdot \vec{i}+\left(\frac{\partial A_{x}}{\partial z}-\frac{\partial A_{z}}{\partial x}\right) \cdot \vec{j}+\left(\frac{\partial A_{y}}{\partial x}-\frac{\partial A_{x}}{\partial y}\right) \cdot \vec{k}
$$

The gradient part $\partial N_{j} / \partial h(h=x, y, z)$ is obtained by the following gradient smoothing operation

$$
\frac{\partial N_{j}}{\partial h}=\int_{\Omega_{s c}} \frac{\partial N_{j}}{\partial h} \Phi_{c}(X) \mathrm{d} \Omega \quad h=(x, y, z)
$$

in which $\Phi_{c}$ is a given smoothing function [33] defined as

$$
\Phi_{c}(X)=\left\{\begin{array}{cc}
1 / V_{s c}, & X \in \Omega_{s c} \\
0, & X \notin \Omega_{s c}
\end{array}\right.
$$

Applying the divergence theorem, one can get the smoothed gradient of domain $\Omega_{s c}$

$$
\begin{gathered}
\frac{\partial N_{j}}{\partial h}=\int_{\Omega_{s c}} \frac{\partial N_{j}}{\partial h} \cdot \frac{1}{V_{s c}} d \Omega=\frac{1}{V_{s c}} \int_{\Omega_{s c}} \frac{\partial N_{j}}{\partial h} d \Omega=\frac{1}{V_{s c}} \int_{\Gamma_{s c}} n_{h}(x, y, z) N_{j} d \Gamma= \\
\sum_{m=1}^{5} \frac{1}{V_{s c}} n_{m h}(x, y, z) N_{j} A_{m} \quad(h=x, y, z)
\end{gathered}
$$

where $\Gamma_{s c}$ is the boundary face of the domain $\Omega_{s c}$, and $n_{m h}(x, y, z)$ is the outward normal vector matrix on boundary face $\Gamma_{s c}$. Note that as only the shape function itself is used to calculate the gradient part, very simple shape functions as shown in 
Fig. 1 can be utilized at integration point on the faces of a cell. Terms of the electromagnetic stiffness matrix can be calculated in the two smoothing cells, for instance,

$$
\int_{\Omega} N_{i, r} \cdot N_{j, t} \mathrm{~d} \Omega=\sum_{s c=1}^{2}\left(N_{i, r}\right)_{s c} \cdot\left(N_{j, t}\right)_{s c} \cdot V_{s c} \quad(r, t=x, y, z)
$$

Substituting Eq. (12) into Eq. (13), it turn out to be

$$
\begin{gathered}
\int_{\Omega} N_{i, r} \cdot N_{j, t} \mathrm{~d} \Omega=\sum_{s c=1}^{2}\left(\sum_{m=1}^{5} \frac{1}{V_{s c}} n_{m r}(x, y, z) N_{i} A_{m}\right) \cdot\left(\sum_{m=1}^{5} \frac{1}{V_{s c}} n_{m t}(x, y, z) N_{j} A_{m}\right) \cdot V_{s c} \\
(r, t=x, y, z)
\end{gathered}
$$

In this way, all items of Eqs. (6) and (8) can be obtained by "Triprism" element.

\subsubsection{Stable nodal integration method (SNIM)}

Stable nodal integration method (SNIM) was proposed by Feng et al. [27] for solid mechanics and extended to acoustic analysis by Wang et al. [34], and has shown the excellent properties in these problems. In this work, it is further extended to electromagnetic analysis in complicated coil and air region.

In the SNIM, the coil and air domain $\Omega^{\prime}$ can be firstly discretized into a set of tetrahedron element, then further subdivided into several non-overlapping smoothing domain $\Omega_{k}$ centered by node $k$ based on the background tetrahedron meshes, as shown in Fig. 2. Linear interpolation is adopted to approximate the field variables in each element. The gradient components of each smoothing domain shown in Eq. (9) can be given by

$$
\frac{\partial N_{j}}{\partial h}=\frac{1}{V_{k}} \int_{\Omega_{k}} \frac{\partial N_{j}}{\partial h} d \Omega=\frac{1}{V_{k}} \int_{\Gamma_{k}} N_{j} \cdot n_{h}(x, y, z) d \Gamma \quad(h=x, y, z)
$$

in which $V_{k}$ is the volume of smoothing domain $\Omega_{k}$. And as linear magnetic field is used over boundary face $\Gamma_{k}$, Eq. (15) can be written as

$$
\frac{\partial N_{j}}{\partial h}=\frac{1}{V_{k}} \sum_{m=1}^{N_{f}} N_{j}\left(x_{m}^{G P}, y_{m}^{G P}, z_{m}^{G P}\right) n_{m h} A_{m} \quad(\mathrm{~h}=x, y, z)
$$

in which $\left(x_{m}^{G P}, y_{m}^{G P}, z_{m}^{G P}\right)$ is the centroid of the boundary patch, $N_{f}$ is the total number of faces of boundary $\Gamma_{k}$, and $n_{m h}, A_{m}$ denote outward unit normal and area of the boundary patch, respectively. 
In order to cure the instability of traditional nodal integration method and improve the accuracy of numerical modeling, a stabilization term is employed here to obtain electromagnetic stiffness matrix. The smoothing domain $\Omega_{k}$ is approximated as a sphere domain $\Omega_{k}^{c}$ with the same volume $V_{k}$. The sphere domain $\Omega_{k}^{c}$ is then subdivided into six equal sub-domains, and the selected integration points $g_{i}^{n}(i=1,2,3,4,5,6)$ lie in $x$-axis, $y$-axis and $z$-axis and keep the same distance $l_{c}$ to node $k$, as layout Fig. 3.

The radius of the approximate sphere domain is expressed as

$$
l_{c}=\sqrt[3]{3 V_{k} /(4 \pi)}
$$

The gradient parts $\partial N_{j} / \partial h(h=x, y, z)$, named as $D N$ for convenience, in $\Omega_{k}^{c}$ is continuous and derivable at the first order, its Taylor expansion can be expressed as

$$
D N=D N_{k}+\frac{\partial D N}{\partial x}\left(x-x_{k}\right)+\frac{\partial D N}{\partial y}\left(y-y_{k}\right)+\frac{\partial D N}{\partial z}\left(z-z_{k}\right)
$$

So $D N$ of the six sub-domains $D N_{1}^{c}, D N_{2}^{c}, D N_{3}^{c}, D N_{4}^{c}, D N_{5}^{c}$, and $D N_{6}^{c}$ are expressed as

$$
\begin{aligned}
& D N_{1}^{c}=D N_{k}-\frac{\partial D N}{\partial x} l_{c}, D N_{2}^{c}=D N_{k}-\frac{\partial D N}{\partial y} l_{c}, D N_{3}^{c}=D N_{k}-\frac{\partial D N}{\partial z} l_{c} \\
& D N_{4}^{c}=D N_{k}-\frac{\partial D N}{\partial x} l_{c}, D N_{5}^{c}=D N_{k}-\frac{\partial D N}{\partial y} l_{c}, D N_{6}^{c}=D N_{k}-\frac{\partial D N}{\partial z} l_{c}
\end{aligned}
$$

Items of the stiffness matrix can be calculated at six integration points, for instance,

$$
\int_{\Omega_{k}^{c}} N_{i, r} \cdot N_{j, t} \mathrm{~d} \Omega=\sum_{m=1}^{6}\left(\left(N_{i, r}\right)_{m} \cdot\left(N_{j, t}\right)_{m}\right) \cdot \frac{V_{k}}{6} \quad(r, t=x, y, z)
$$

Substituting Eq. (19) into Eq. (20), it turns out to be

$$
\begin{gathered}
\int_{\Omega_{k}^{c}} N_{i, r} \cdot N_{i, t} \mathrm{~d} \Omega=(D N(r) \cdot D N(t))_{k} \cdot V_{k}+\Delta D N(r)_{x} \cdot \Delta D N(t)_{x} \cdot \frac{V_{k}}{3}+ \\
\Delta D N(r)_{y} \cdot \Delta D N(t)_{y} \cdot \frac{V_{k}}{3}+\Delta D N(r)_{z} \cdot \Delta D N(t)_{z} \cdot \frac{V_{k}}{3}
\end{gathered}
$$

where 


$$
\Delta D N_{x}=\frac{\partial D N}{\partial x} \cdot l_{c}, \Delta D N_{y}=\frac{\partial D N}{\partial y} \cdot l_{c}, \Delta D N_{z}=\frac{\partial D N}{\partial z} \cdot l_{c}
$$

\section{Formulations for mechanical field}

In mechanical field, shell element is employed to analyze explicit nonlinear dynamic process, the finite element equation can be written as

$$
\mathbf{M u ̈}=\mathbf{f}^{e x t}-\mathbf{f}^{\mathrm{int}}
$$

where $\mathbf{M}$ is the mass matrix, $\mathbf{f}^{\text {ext }}$ and $\mathbf{f}^{\text {int }}$ are the nodal force vectors arising from external forces and internal element resistances, respectively. Lorentz force on sheet metal, contact and friction force (if exits) are considered as the external forces in mechanical field of EMF process. So the external force can be expressed as

$$
\mathbf{f}^{e x t}=\sum \iint \mathbf{N}^{\mathrm{T}}\left(\mathbf{J}_{e} \times \mathbf{B}\right) \mathrm{d} \Omega+\left(\mathbf{f}_{n}+\mathbf{f}_{\tau}\right)
$$

in which $\mathbf{J}_{e}=-\sigma \partial \mathbf{A} / \partial t$ is the induced eddy current flows through the sheet metal, $\mathbf{f}_{n}$ and $\mathbf{f}_{\tau}$ denote contact force vector (along tool normal direction) and friction force vector (along tool tangential direction) respectively.

\subsection{Basic formulation for triangular shell element}

A three-node triangular facet shell element is utilized here. Fig. 4 shows an embedded element coordinate system defined in terms of the 3 nodal coordinates, which deforms with the element. The local $x$-axis $\hat{x}\left(\overrightarrow{\mathbf{e}}_{1}\right)$ is directed from node 1 to node 2 , and the element's normal axis $\hat{z}\left(\overrightarrow{\mathbf{e}}_{3}\right)$ is defined by the vector cross product of a vector along $\hat{x}$ with a vector constructed from node 1 to node 3 . The local $y$-axis $\hat{y}\left(\overrightarrow{\mathbf{e}}_{2}\right)$ is defined by the base unit vector product $\overrightarrow{\mathbf{e}}_{3}$ with $\overrightarrow{\mathbf{e}}_{1}$.

Based on the first-order shear deformation theory of plate, the velocity at arbitrary point of shell in Cartesian coordinate system can be expressed as

$$
\mathbf{v}=\mathbf{v}^{m}-\hat{z} \overrightarrow{\mathbf{e}}_{3} \times \mathbf{w}
$$

where $\mathbf{v}^{m}$ is the velocity of the mid-surface, $\mathbf{w}$ is the angular velocity vector, and $\hat{z}$ is the distance along the fiber direction (thickness) of the shell element. The corresponding co-rotational components of the velocity strain are given by 


$$
\hat{d}_{i j}=\frac{1}{2}\left(\frac{\partial \hat{v}_{i}}{\partial \hat{x}_{j}}+\frac{\partial \hat{v}_{j}}{\partial \hat{x}_{i}}\right)
$$

Standard linear interpolation with shape function $N_{I}(\hat{x}, \hat{y})$ of triangular element is adopted to evaluate the nodal values, the interpolation relations are given by

$$
\hat{v}^{m}=\sum_{I=1}^{3} N_{I}(\hat{x}, \hat{y}) \hat{v}_{I}, \quad \hat{w}^{m}=\sum_{I=1}^{3} N_{I}(\hat{x}, \hat{y}) \hat{w}_{I}
$$

Transformation matrix is defined between local components and global components, for instance, $\hat{\mathbf{v}}_{I}^{m}=\left\{\begin{array}{lll}\hat{v}_{I x} & \hat{v}_{I y} & \hat{v}_{I z}\end{array}\right\}^{\mathbf{T}}$ and $\quad \mathbf{v}_{I}^{m}=\left\{\begin{array}{lll}v_{I x} & v_{I y} & v_{I z}\end{array}\right\}^{\mathbf{T}}$, and it defined as

$$
\mathbf{v}_{I}^{m}=\left\{\begin{array}{l}
v_{I x} \\
v_{I y} \\
v_{I z}
\end{array}\right\}=\left[\begin{array}{lll}
c_{\hat{x} x} & c_{\hat{y} x} & c_{\hat{z} x} \\
c_{\hat{x} y} & c_{\hat{y} y} & c_{\hat{z} y} \\
c_{\hat{x} z} & c_{\hat{y} z} & c_{\hat{z} z}
\end{array}\right]\left\{\begin{array}{l}
\hat{v}_{I x} \\
\hat{v}_{I y} \\
\hat{v}_{I z}
\end{array}\right\}=\mathbf{T} \cdot \hat{\mathbf{v}}_{I}^{m}
$$

in which as usual $c_{\hat{x} x}$ is the cosine between the $\hat{x}$ and $x$ axes, and so on. Substituting Eq. (25) into Eq, (26) and according to the interpolation relations, the in-plane velocity strain vector can be written in terms of the middle plane deformation as below

$$
\hat{\mathbf{d}}^{I n}=\left\{\begin{array}{c}
\hat{d}_{x x} \\
\hat{d}_{y y} \\
2 \hat{d}_{x y}
\end{array}\right\}=\sum_{I=1}^{3} \hat{\mathbf{B}}_{I}^{m} \hat{v}_{I}^{\prime}+z \sum_{I=1}^{3} \hat{\mathbf{B}}_{I}^{b} \hat{v}_{I}^{\prime \prime}
$$

where $\hat{\mathbf{B}}_{I}^{m}$ and $\hat{\mathbf{B}}_{I}^{b}$ denote the membrane and bending part of gradient matrix, respectively,

$$
\hat{\mathbf{B}}_{I}^{m}=\left[\begin{array}{cc}
\frac{\partial N_{I}}{\partial \hat{x}} & 0 \\
0 & \frac{\partial N_{I}}{\partial \hat{y}} \\
\frac{\partial N_{I}}{\partial \hat{y}} & \frac{\partial N_{I}}{\partial \hat{x}}
\end{array}\right], \quad \hat{\mathbf{B}}_{I}^{b}=\left[\begin{array}{ccc}
0 & 0 & \frac{\partial N_{I}}{\partial \hat{x}} \\
0 & -\frac{\partial N_{I}}{\partial \hat{y}} & 0 \\
0 & -\frac{\partial N_{I}}{\partial \hat{x}} & \frac{\partial N_{I}}{\partial \hat{y}}
\end{array}\right], \quad \hat{v}_{I}^{\prime}=\left\{\begin{array}{c}
\hat{v}_{x I} \\
\hat{v}_{y I}
\end{array}\right\}, \quad \hat{v}_{I}^{\prime \prime}=\left\{\begin{array}{c}
\hat{v}_{z I} \\
\hat{w}_{x I} \\
\hat{w}_{y I}
\end{array}\right\}
$$

For shear velocity strain, shear locking is emerged in the Mindlin-Reissner plate when thickness is decreased overly. To eliminate the shear locking [35, 36], well known "Discrete Shear Gap" (DSG) method developed by Bletzinge et al. [28] , is introduced here. In each triangular element, the transverse shear velocity strain can be given as 


$$
2 \hat{\mathbf{d}}_{x z}=\sum_{I=1}^{3} \frac{\partial N_{I}}{\partial \hat{x}} \Delta \dot{w}_{\hat{x} I}+\sum_{I=1}^{3} \frac{\partial N_{I}}{\partial \hat{x}} \Delta \dot{w}_{\hat{y} I}, \quad 2 \hat{\mathbf{d}}_{y z}=\sum_{I=1}^{3} \frac{\partial N_{I}}{\partial \hat{y}} \Delta \dot{w}_{\hat{x} I}+\sum_{I=1}^{3} \frac{\partial N_{I}}{\partial \hat{y}} \Delta \dot{w}_{\hat{y} I}
$$

in which $\Delta \dot{w}_{\hat{x} I}$ and $\Delta \dot{w}_{\hat{y} I}$ are the rate of discrete shear gaps at node $I$, they are given as

$$
\begin{aligned}
& \Delta \dot{w}_{\hat{x} 1}=\Delta \dot{w}_{\hat{x} 3}=\Delta \dot{w}_{\hat{y} 1}=\Delta \dot{w}_{\hat{y} 3}=0 \\
& \Delta \dot{w}_{\hat{x} 2}=\left(\hat{v}_{z 2}-\hat{v}_{z 1}\right)+\frac{1}{2} \hat{x}_{2}\left(\hat{w}_{x 1}+\hat{w}_{x 2}\right)+\frac{1}{2} \hat{y}_{2}\left(\hat{w}_{y 1}+\hat{w}_{y 2}\right) \\
& \Delta \dot{w}_{\hat{y} 2}=\left(\hat{v}_{z 3}-\hat{v}_{z 1}\right)+\frac{1}{2} \hat{x}_{3}\left(\hat{w}_{x 1}+\hat{w}_{x 3}\right)+\frac{1}{2} \hat{y}_{2}\left(\hat{w}_{y 1}+\hat{w}_{y 3}\right)
\end{aligned}
$$

From the above Eqs. (31) and (32), the shear velocity strain can be written by the following matrix form

$$
\hat{\mathbf{d}}^{s}=\left\{\begin{array}{l}
2 \hat{d}_{x z} \\
2 \hat{d}_{y z}
\end{array}\right\}=\hat{\mathbf{B}}_{I}^{s} \hat{v}_{I}^{\prime \prime}
$$

where

$$
\begin{gathered}
\hat{\mathbf{B}}_{1}^{s}=\frac{1}{2 A_{e}}\left[\begin{array}{ccc}
\hat{y}_{2}-\hat{y}_{3} & 0 & A_{e} \\
\hat{x}_{3}-\hat{x}_{2} & -A_{e} & 0
\end{array}\right], \hat{\mathbf{B}}_{2}^{s}=\frac{1}{2 A_{e}}\left[\begin{array}{ccc}
\hat{y}_{3} & -\hat{y}_{2} \hat{y}_{3} / 2 & \hat{x}_{2} \hat{y}_{3} / 2 \\
-\hat{x}_{3} & \hat{y}_{2} \hat{x}_{3} / 2 & -\hat{x}_{2} \hat{x}_{3} / 2
\end{array}\right], \\
\hat{\mathbf{B}}_{3}^{s}=\frac{1}{2 A_{e}}\left[\begin{array}{ccc}
-\hat{y}_{2} & \hat{y}_{2} \hat{y}_{3} / 2 & -\hat{y}_{2} \hat{x}_{3} / 2 \\
\hat{x}_{2} & -\hat{x}_{2} \hat{y}_{3} / 2 & \hat{x}_{2} \hat{x}_{3} / 2
\end{array}\right]
\end{gathered}
$$

\subsection{Stress-strain law and internal force vector}

We consider the shell in a state of plane stress, thus, the stresses are composite of in-plane and shear parts

$$
\hat{\boldsymbol{\sigma}}=\left\{\begin{array}{l}
\hat{\boldsymbol{\sigma}}^{I n} \\
\hat{\boldsymbol{\sigma}}^{s}
\end{array}\right\}, \quad \hat{\boldsymbol{\sigma}}^{I n}=\left\{\begin{array}{lll}
\sigma_{x x} & \sigma_{y y} & \sigma_{x y}
\end{array}\right\}^{\mathrm{T}}, \hat{\boldsymbol{\sigma}}^{s}=\left\{\begin{array}{ll}
\sigma_{x z} & \sigma_{y z}
\end{array}\right\}^{\mathrm{T}}
$$

For accurate prediction of the gradual spread of plasticity in shell, a layered approach is utilized in which the shell thickness is subdivided into a chosen number of layers. The yield criteria is presented in terms of the stresses corresponding to each layer. At each layer, the stress state is represented by values associated to the middle of the layer.

Given the set $\left(\hat{\boldsymbol{\sigma}}_{n}, \hat{\boldsymbol{\varepsilon}}_{n}\right)$ at time $t_{n}$ and and the strain increment $\Delta \hat{\boldsymbol{\varepsilon}}=\int_{t_{n}}^{t_{n+1}} \hat{\mathbf{d}} d t$ in each layer, the strain at $t_{n+1}$ can be given by 


$$
\hat{\boldsymbol{\varepsilon}}_{n+1}=\hat{\boldsymbol{\varepsilon}}_{n}+\Delta \hat{\boldsymbol{\varepsilon}}
$$

and the stresses $\hat{\boldsymbol{\sigma}}_{n+1}^{I n}$ and $\hat{\boldsymbol{\sigma}}_{n+1}^{s}$ at time $t_{n+1}$ can be computed by the following equations

$$
\hat{\boldsymbol{\sigma}}_{n+1}^{I n}=\hat{\boldsymbol{\sigma}}_{n}^{I n}+\Delta \hat{\boldsymbol{\sigma}}^{I n}=\mathbf{D}^{e}\left(\Delta \hat{\boldsymbol{\varepsilon}}^{I n}-\left(\Delta \hat{\boldsymbol{\varepsilon}}^{I n}\right)^{\mathrm{p}}\right), \quad \hat{\boldsymbol{\sigma}}_{n+1}^{s}=\hat{\boldsymbol{\sigma}}_{n}^{s}+\mathbf{D}^{s} \Delta \hat{\boldsymbol{\varepsilon}}^{s}
$$

where $\left(\Delta \hat{\boldsymbol{\varepsilon}}^{I n}\right)^{\mathrm{p}}$ is the incremental of in-plane plastic strain, and $\mathbf{D}^{e}$ and $\mathbf{D}^{s}$ denote elastic and transverse shear constitutive matrix respectively.

$$
\mathbf{D}^{e}=\frac{E}{1-v^{2}}\left[\begin{array}{ccc}
1 & v & 0 \\
v & 1 & 0 \\
0 & 0 & \frac{1-v}{2}
\end{array}\right], \mathbf{D}^{s}=\kappa\left[\begin{array}{cc}
G & 0 \\
0 & G
\end{array}\right]
$$

in which $E$ is Young's modulus, $v$ is Poisson's ratio, $\kappa$ is the shear correction factor and $G$ is the shear modulus.

It is assumed that the Von-Mises yield criterion is valid in this work which can be presented as

$$
f=\frac{1}{2} s_{i j} s_{i j}-\frac{1}{3} \sigma_{s}^{2}\left(\bar{\varepsilon}^{p}\right) \leq 0
$$

where $s_{i j}$ is the deviatoric part of the stress, $\sigma_{s}\left(\bar{\varepsilon}^{p}\right)$ is the present-time yield stress, which is a function of the equivalent plastic strain $\bar{\varepsilon}^{p} \cdot \sigma_{s}\left(\bar{\varepsilon}^{p}\right)$ can be written as

$$
\sigma_{s}\left(\bar{\varepsilon}^{p}\right)_{n+1}=\sigma_{s}\left(\bar{\varepsilon}^{p}\right)_{n}+H^{\prime} \bar{\varepsilon}^{p}
$$

where $H^{\prime}=d \sigma_{s} / d \bar{\varepsilon}^{p}$ is plastic module of the material.

According to Eq. (44), if the associative rule is considered then

$$
d_{i j}^{p}=d \lambda \frac{\partial f}{\partial \sigma_{i j}}=d \lambda \frac{\partial f}{\partial s_{i j}}
$$

in the above equation $d \lambda$ is the plastic consistency parameter. And Loading -unloading conditions are given by

$$
d \lambda \geq 0, \quad f \leq 0, d \lambda \cdot f=0
$$

By applying a load increment, the status of a layer may or may not change from the elastic state to the plastic state. In case of the former, the updated stress vector should satisfy the second consistency, the radial return mapping algorithm $[37,38]$ is 
used in the present work to return the stresses to the yield surface. For high strain rate material under impact loads, strain rate should be considered in the constitutive equation, and plastic module becomes: $H^{\prime}=\partial \sigma_{s} / \partial \bar{\varepsilon}^{p}+\partial \sigma_{s} / \partial \Delta \bar{\varepsilon}^{p}$. However, it unstable in one time step result from the effect of strain rate. In this work, a stable dichotomy iteration method [39] is employed here to find the real plastic module.

After suitable constitutive evaluation through the above process, the resulting local stresses are integrated through the thickness of the shell to obtain local forces and moments. The nodal internal force vector is given by

$$
f_{I}^{\mathrm{int}}=\left\{\begin{array}{lllll}
\hat{f}_{x I}^{\mathrm{int}} & \hat{f}_{y I}^{\mathrm{int}} & \hat{f}_{z I}^{\mathrm{int}} & \hat{m}_{x I}^{\mathrm{int}} & \hat{m}_{y I}^{\mathrm{int}}
\end{array}\right\}^{\mathrm{T}}
$$

where

$$
\left\{\begin{array}{l}
\hat{f}_{x I}^{\text {int }} \\
\hat{f}_{y I}^{\text {int }}
\end{array}\right\}=A_{e}\left(\hat{\mathbf{B}}_{I}^{m}\right)^{\mathrm{T}}\left\{\begin{array}{l}
\hat{n}_{x} \\
\hat{n}_{y} \\
\hat{n}_{x y}
\end{array}\right\},\left\{\begin{array}{l}
\hat{f}_{z I}^{\mathrm{int}} \\
\hat{m}_{x I}^{\mathrm{int}} \\
\hat{m}_{y I}^{\mathrm{int}}
\end{array}\right\}=A_{e}\left(\hat{\mathbf{B}}_{I}^{b}\right)^{\mathrm{T}}\left\{\begin{array}{l}
\hat{r}_{x} \\
\hat{r}_{y} \\
\hat{r}_{x y}
\end{array}\right\}+A_{e}\left(\hat{\mathbf{B}}_{I}^{s}\right)^{\mathrm{T}}\left\{\begin{array}{l}
\hat{n}_{x z} \\
\hat{n}_{y z}
\end{array}\right\}
$$

the generalized stress is defined as

$$
\begin{gathered}
\hat{n}_{x}=\int_{-t / 2}^{t / 2} \hat{\sigma}_{x} \mathrm{~d} z, \quad \hat{n}_{y}=\int_{-t / 2}^{t / 2} \hat{\sigma}_{y} \mathrm{~d} z, \quad \hat{n}_{x y}=\int_{-t / 2}^{t / 2} \hat{\sigma}_{x y} \mathrm{~d} z \\
\hat{r}_{x}=\int_{-t / 2}^{t / 2} z \hat{\sigma}_{x} \mathrm{~d} z, \quad \hat{r}_{y}=\int_{-t / 2}^{t / 2} z \hat{\sigma}_{y} \mathrm{~d} z, \quad \hat{r}_{x y}=\int_{-t / 2}^{t / 2} z \hat{\sigma}_{x y} \mathrm{~d} z \\
\hat{n}_{x z}=\int_{-t / 2}^{t / 2} \hat{\sigma}_{x z} \mathrm{~d} z, \quad \hat{n}_{y z}=\int_{-t / 2}^{t / 2} \hat{\sigma}_{y z} \mathrm{~d} z
\end{gathered}
$$

\subsection{Contact problem}

The numerical simulation for complicated EMF process customarily requires the definition of the frictional contact conditions between the forming tools and sheet metal. In this work, the formulation for 3D contact problems is evaluated by using penalty method and Coulomb friction law. Considering a two-body frictional contact problem, as illustrated in Fig. 5.

The penetration $p$ of any node into the tool surface is defined by

$$
\mathbf{f}_{n}=-\alpha_{n} p \cdot \vec{n}
$$

where $\alpha_{n}$ is a chosen penalty factor, $\vec{n}$ is the tool normal direction.

Assuming the Coulomb's friction law at the contact interface, the contact constrains over the tangential direction associated with the friction law are given as follows:

$$
\overline{\mathbf{f}_{\tau}}=\mathbf{f}_{\tau}^{n}+\left(-E_{f} \Delta \mathbf{u}_{\tau}\right)
$$


where, $E_{f}$ is the friction module, and $\Delta \mathbf{u}_{\tau}$ is the relative sliding displacement. Then, if $\left|f_{\tau}\right|+\mu f_{n} \leq 0 \quad$ ( $\mu$ denote the coefficient of friction between the workpiece and tool), and the friction force at time $t_{n+1}$ is updated by Eq. (47). Else, the friction force at time $t_{n+1}$ can be given by

$$
\mathbf{f}_{\tau}^{n+1}=\mu\left|\mathbf{f}_{n}\right| \overline{\mathbf{f}}_{\tau} /\left|\overline{\mathbf{f}}_{\tau}\right|
$$

\section{Interactive mapping between "Triprism" and shell element}

In order to cope with the data interaction between electromagnetic and mechanical field, an interactive mapping between "Triprism" solid and shell element is proposed. Lorentz force on solid evaluated in electromagnetic field is gathered in mid-surface, and then transfered into shell in mechanical field. Considering the effect of sheet geometric deformation in electromagnetic field, the "Triprism" solid element can be dragged over the thickness direction based on the nodal coordinates of deformed shell element. Therefore, in computation of the coupled electromagnetic-mechanical formulation, "Triprism" solid and triangular shell element is interactive mapping with each other at every time step. As shown in the Fig. 6, the mapping scheme is illustrated below.

A nodal relationship $\operatorname{Cn}(I, k)(k=1,2, \cdots m)$ between the two different type elements can be established based on the nodal normal vectors $\vec{n}^{\prime}$ and $\vec{n}$. The nodal Lorentz force in shell element is calculated by the following equation

$$
\overline{\mathbf{f}}_{I}^{L}=\sum_{j=1}^{m} \tilde{\mathbf{f}}_{N(j)}^{L} \quad(N(j)=C n(I, j))
$$

in which $m$ denotes the number of nodes over shell thickness direction in "Triprism" element, the node number and Lorentz force of the node in electromagnetic field which are denoted as $N(j)$ and $\tilde{\mathbf{f}}_{N(j)}^{L}$, respectively.

The Lorentz forces make changes in the geometric configuration, and mechanical deformations, in turn, affect the distribution of flux density and Lorentz force. Consequently, relative large deformation of the sheet is not negligible which affects the electromagnetic field. A dynamic moving mesh technique [40] based on the interactive mapping of "Triprism" solid and triangular shell element is presented here to update the air meshes in electromagnetic field. In this mesh updating scheme, the 
mesh 'flow' is governed by the modified equations of linear elasticity equations without body force and prescribed traction. These equations are solved to determine the internal nodal displacements of the air mesh based on the given displacement of sheet.

In this work, the initial given displacement of sheet metal is based on shell element in mechanical field. According to the nodal relationship between "Triprism" and shell element, the nodal displacement of "Triprism" element $\hat{u}$ can be obtained by making use of the nodal displacement value $u^{s}$ of shell element. The nodal displacements of "Triprism" element can be written as

$$
\hat{u}(C n(I, j))=u^{s}(I)+\vec{n} \cdot\left(-\frac{t}{2}+(j-1) \cdot \frac{t}{m-1}\right) \quad(j=1,2, \cdots m)
$$

in which $t$ is the thickness of the shell, and $j$ represent the $j$-th node of "Triprism" over the thickness direction. $\hat{u}(), u^{s}()$ denote the nodal displacement of "Triprism" and shell element respectively. In this way, the "Triprism" element generation rule is simultaneously established based on triangular shell element.

Consider an elastic body occupying a region $\Omega$ with boundary $\Gamma$ as shown in Fig. 7. The displacement of the mesh is given by $\hat{\mathbf{u}}(x)$ and is governed by the equilibrium equations of elasticity. The strain tensor $\varepsilon$ is directly calculated by

$$
\varepsilon=\frac{1}{2}\left(\nabla \hat{\mathbf{u}}+(\nabla \hat{\mathbf{u}})^{\mathrm{T}}\right)
$$

The stiffness matrix $\mathbf{K}^{e}$ in the local coordinate is defined as

$$
\mathbf{K}^{e}=\int_{\Omega}\left(\mathbf{B}^{T} \mathbf{D}^{e} \mathbf{B J}\right) \mathrm{d} \xi \mathrm{d} \eta \mathrm{d} \varsigma
$$

then, the material constant $\mathbf{D}^{e}$ is redefined as

$$
\tilde{\mathbf{D}}^{e}=\mathbf{J D}^{e}
$$

therefore, the local stiffness matrix becomes

$$
\mathbf{K}^{e}=\int_{\Omega}\left(\mathbf{B}^{T} \frac{\tilde{\mathbf{D}}^{e}}{\mathbf{J}} \mathbf{B} \mathbf{J}\right) \mathrm{d} \xi \mathrm{d} \eta \mathrm{d} \varsigma
$$

It is desirable to retain the structure of the mesh in the more refined areas, and have most of the deformation weighted towards the large element regions of the mesh. Through the mesh updating technique based on "Triprism" element, the air mesh change more regular in the wake of deformation of the sheet. 


\section{Examples}

\subsection{TEAM problem 20}

Benchmark problem 20 for the TEAM Workshop [41] is a 3D nonlinear magneto-static problem, as shown in Fig. 8. This example is employed here to attest the good performance of the proposed "Triprism" element in predicting flux density. Fig. 9 shows the mesh model for problem domain and the positions at which flux densities are measured. Regions of coil, center pole and yoke are meshed with six-node "Triprism" elements (4226 elements for center pole and yoke, and 8040 elements for coil), and region of air is meshed with SNIM four-node tetrahedral elements (6993 nodes and 29039 elements). The $z$-directional component $B_{z}$ of flux density at the mid-point $P 1$ and at the edge $P 2$ in the gap as well as the $B_{z}$ of average flux density in the center pole $S 1(\alpha-\beta)$ and yoke $S 2(\gamma-\delta)$ are listed in Table 1. The results by present mixed "Triprism" and SNIM element are compared with experimental measured results, as illustrated in Fig. 10. The present element generates great results for this problem. Therefore, the mixed meshes of "Triprism" and SNIM element can provide a good prediction of flux density.

\subsection{Tube expansion}

In order to validate the feasibility of present interactive mapping of "Triprism" and shell element and to illustrate the efficiency of shell element in forming process contrast to solid element, a tube free expansion example is employed here. Both solid ("Triprism") and shell element are utilized to solving the dynamic deformation process in mechanical field. The dimension and shape of the tube expansion system are illustrated in Fig. 11. Owing to the symmetry of the system, only $1 / 8$ of the model is employed. A finite element mesh model for electromagnetic and mechanical field is shown in Fig. 12. Six-node "Triprism" elements for tube are used in electromagnetic analysis (6120 elements), and the regions of coil and air are meshed with SNIM tetrahedral elements. Five layers of elements are meshed over the thickness direction considering to the skin effects. The material parameters of the tube expansion system are given in Table 2. The load current [42] flowing in the forming coil is calculated using the following equation 


$$
I(t)=-10.5 \times e^{-1430 t}+1.06 \times e^{-3240 t} \times \sin \left(17800 t+9.90 \times 10^{-4}\right)
$$

According to the work from Suzuki et al. [43], the flow stress of the tube was approximated by:

$$
\sigma / M P a=162 \cdot(\varepsilon+0.000663)^{0.411}
$$

In this EMF simulation process, the time step is $5 \mu$ s and the total simulation time is $350 \mu \mathrm{s}$. As can be seen in Fig. 13, The tendency of center radial displacement of the tube shows agreement with experimental one. The radial displacement of this work is $5.92 \mathrm{~mm}$, while the experimental one is $6.00 \mathrm{~mm}$ at the same time. A good agreement is obtained between the present work and experimental results. The final displacement of the tube is shown in Fig. 14. The present results by shell and solid ("Triprism") element all have great agreement with the experimental one, and better than the earlier approach. Therefore, the present method can better predict the electromagnetic forming process. The changes of tube shape at different times are shown in Fig. 15, and deformation completes at $300 \mu$ s. Fig. 16 shows the deformed shape of tube by shell element and solid element, respectively. Computational time of the two type element employed in the work solving the forming process is illustrated in Fig. 17, the modeling with application of shell element in mechanical field facilitate a large reduction (about 80 times) of computational cost.

\subsection{Sheet metal free bulging}

A sheet metal free bulging example is employed here to further illustrate the performance of present interaction mapping element. The geometry and detail dimension of electromagnetic forming system is shown in Fig. 18. A six turn flat spiral coil is used to deform the sheet metal by magnetic force. Only $1 / 4$ of the model is used due to the symmetry of system. A finite element mesh for electromagnetic and mechanical field is shown in Fig. 19. Similarly, the sheet region is meshed with the proposed six-node "Triprism" element (4535 elements), and five layers of elements are used over thickness direction owing to the skin effects. The regions of coil, as well as air surrounding the tube, are meshed with SNIM four-node tetrahedral elements. In mechanical field, only sheet meshed with shell element is taken into account. During the forming process, the holder and binder were considered to be fixed, and contacts are considered between the die and sheet as well as the sheet and holder. Coulomb friction law is used with a friction coefficient of 0.25 . 
The simulation load current $I(t)$ flowing in the coil is approximately described by the following equation

$$
I(t)=U \sqrt{\frac{C}{L}} e^{-\beta \cdot t} \sin (\omega t)
$$

where $U=9 k V$, represents the initial discharge voltage; $C=100 \mu F$ is the total capacitance of capacitor bank; $L=1.277 \mu H$ is the equivalent inductance of the system; $\beta=165.79 \mathrm{~s}^{-1}$ is the damping coefficient of circuit; $\omega=4366 \mathrm{~Hz}$ is the angular frequency. The aluminum alloy AA3003 sheet with $1.0 \mathrm{~mm}$ in thickness is used, and material parameters of the system are listed in Table 3. Considering to the effect of strain rate, the constitutive behavior of the sheet can be seen in Eq. (58)

$$
\sigma / M p a=\sigma_{y}\left[1+\left(\frac{\dot{\varepsilon}}{P}\right)^{m}\right]
$$

in which $\sigma_{y} / M p a=138.0 \times(\varepsilon+0.0006)^{0.137}$ is the quasi-static stress; $\varepsilon$ is the plastic strain and $\dot{\varepsilon}$ is the strain rate. $p=6500 \mathrm{~s}^{-1}, m=0.25$ are the plastic strain rate parameter.

The final displacement over the radial direction is plotted in Fig. 20, and simulation results predicted the deformation. Fig. 21 shows the deformation of sheet at different time. Results illustrated that the present interactive mapping element performs well in the sheet metal free bulging analysis.

\subsection{Sheet metal forming with a middle-block die}

Finally, a non-axisymmetric electromagnetic forming example is employed. This example is a two-step forming scheme developed by Noh et al. [44]. Here, the two-step forming process is simulated by the present interactive mapping element. The process of using a middle-block die for sheet metal forming is illustrated in Fig. 22. Dynamic moving mesh technique is adopted in the analysis to update the air meshes based on the sheet deformation. The morphing region of electromagnetic field, including the sheet and air surrounding it, is meshed with "Triprism" element (7854 elements). Fig. 23 illustrated a mesh model in the analysis. The load current with $U=9 k V$ and $U=13 k V$ for the two forming step is plotted in Fig. 24. The forming system material properties are listed in Table 4. An Al-1100-O sheet with thickness $1.27 \mathrm{~mm}$ is used for EMF process, and the isotropic hardening constitutive model is 
adopted with strain rate dependency controlled by Cowper-Symonds rate dependent power law. The formulation can be expressed as Eq. (58), and the quasi-static data is $\sigma_{y}=180.47 \dot{\varepsilon}^{0.20}$ as well as the strain rate parameters for Al-1100-O are $P=6500$, $m=0.25$. Coulomb friction law used with a friction coefficient of 0.25 is defined between the sheet and mold.

A seven-turn spiral coil with a section area of $3 \mathrm{~mm} \times 10 \mathrm{~mm}$ and inner radius of $25 \mathrm{~mm}$ is used to deform the sheet firstly. After preforming, a six-turn coil with inner radius of $10 \mathrm{~mm}$ is employed to match the required shape. Fig. 23 shows updating of the air meshes based on the sheet deformation in mechanical field, "Triprism" element used in the morphing region is highly effective in preventing extreme distortion of element and maintaining the overall mesh quality. The displacements along line A-A' (shown in Fig. 24) of the two step forming process are compared with the experimental ones, as shown in Fig. 25. The simulation results agree well with the experiment for first step, and can well predict the deforming tendency for all the two step forming. Fig. 26 shows the deformation of sheet metal at different time. In the two-step strategy, there is no large bounce on the sheet. This example confirmed that the present interactive mapping of "Triprism" and triangular shell element applied in non-axisymmetric parts of EMF process is reliable.

\section{Conclusions}

In contrast to conventional approach which solid elements (tetrahedron and hexahedron) were constantly used in mechanical field of EMF process, a novel approach with interactive mapping of triangular prism solid and shell element for solving coupled magnetic-structural analysis of EMF process is proposed. Several EMF process examples are implemented, and the results by present modeling agree well with the experiment data. Following features of the proposed approach have been confirmed:

(1) The proposed six-node "Triprism" element can accurately predict the flux density and that is effective for electromagnetic analysis.

(2) Shell element employed in mechanical field for dynamic elasto-plasticity analysis is feasible, and facilitating a large reduction of computational costs without significant loss of accuracy. 
(3) Including the "Triprism" generation rule and nodal relationship are simultaneously established based on the geometry of triangular shell element, interactive mapping of “Triprism" solid and shell element is reliable in these EMF process.

(4) Dynamic moving mesh method makes the air mesh change more regular in the wake of deformation of the sheet since the air region is divided into six-node “Triprism” element.

(5) The present approach with interactive mapping of "Triprism" solid and shell element applied in EMF process with complex structure of industrial applications is feasible.

\section{Acknowledgments}

The support of National Science Foundation of China (11472101), State Key Program of National Natural Science of China (61232014), and Hunan Provincial Natural Science Foundation of China (2015JJ3037) are gratefully acknowledged.

\section{References}

1. V. Psyk, D. Risch, B.L. Kinsey, A.E. Tekkaya, M. Kleiner, Electromagnetic forming - A review, J. Mater. Process. Technol. 211 (2011) 787-829.

2. D. Gayakwad, M.K. Dargar, P.K. Sharma, R. purohit, R.S. Rana, A Review on Electromagnetic Forming Process, Procedia Mater. Sci. 6 (2014) 520-527.

3. A. Masserey, J. Rappaz, R. Rozsnyo, Numerical integration of the three-dimensional Green kernel for an electromagnetic problem, J. Comput. Phys. 205 (1) (2005) 48-71.

4. S.J. Cooke, R. Shtokhamer, A.A. Mondelli, B. Levush, A finite integration method for conformal, structured-grid, electromagnetic simulation, J. Comput. Phys. 215 (2006) 321-347.

5. Z.H. Li, Q. Ma, J. Cui, Second-order two-scale finite element algorithm for dynamic thermo-mechanical coupling problem in symmetric structure, J. Comput. Phys. 314 (2016) 712-748. 
6. S. May, M. Kästner, S. Müller, V. Ulbricht, A hybrid IGAFEM/IGABEM formulation for two-dimensional stationary magnetic and magneto-mechanical field problems. Comput. Methods Appl. Mech. Engrg. 273 (1) (2014) 161-180.

7. P. G. Maginot, J. C. Ragusa, J. E. Morel, High-order solution methods for grey discrete ordinates thermal radiative transfer, J. Comput. Phys. 327 (2016) 719-746.

8. M.P. Errera, S. Chemin, Optimal solutions of numerical interface conditions in fluid-structure thermal analysis, J. Comput. Phys. 245 (2013) 431-455.

9. R. Otin, A numerical model for the search of the optimum frequency in electromagnetic metal forming. Int. J. Solids. Struct. 50 (10) (2013) 1605-1612.

10. M. Stiemer, J. Unger, B. Svendsen, Algorithmic formulation and numerical implementation of coupled multifield models for electromagnetic metal forming simulations, Int. J. Numer. Meth. Eng, 68 (13) (2006) 1301-1328.

11. N. Takatsu, M. Kato, K. Sato, T. Tobe, High-speed forming of metal sheets by electromagnetic force, JSME Int. Journal. Ser. 3, Vib. Control Eng. Eng. Ind. 31 (1988) 142-148.

12. G.K. Fenton, G.S. Daehn, Modeling of electromagnetically formed sheet metal, J. Mater. Process. Technol. 75 (1998) 6-16.

13. A.G. Mamalis, D.E. Manolakos, A.G. Kladas, A.K. Koumoutsos, Electromagnetic Forming Tools and Processing Conditions: Numerical Simulation, Mater. Manuf. Process. 21 (2006) 411-423.

14. H.P. Yu, C.F. Li, J.H. Deng, Sequential coupling simulation for electromagnetic-mechanical tube compression by finite element analysis, J. Mater. Process. Technol. 209 (2009) 707-713.

15. X.H. Cui, J.H. Mo, J. Li, L. Huang, Y. Zhu, Z.W. Li, K. Zhong, Effect of second current pulse and different algorithms on simulation accuracy for electromagnetic sheet forming, Int. J. Adv. Manuf. Technol. 68 (2013) 1137-1146.

16. G. Bartels, W. Schätzing, H.-P. Scheibe, M. Leone, Comparison of two different simulation algorithms for the electromagnetic tube compression, Int. J. Mater. Form. 2 (2009) 693-696. 
17. M.A. Bahmani, K. Niayesh, A. Karimi, 3D Simulation of magnetic field distribution in electromagnetic forming systems with field-shaper, J. Mater. Process. Technol. 209 (2009) 2295-2301.

18. D.A. Oliveira, M.J. Worswick, M. Finn, D. Newman, Electromagnetic forming of aluminum alloy sheet: Free-form and cavity fill experiments and model, J. Mater. Process. Technol. 170 (2005) 350-362.

19. X.H. Cui, J.H. Mo, F. Han, 3D Multi-physics field simulation of electromagnetic tube forming, Int. J. Adv. Manuf. Technol. 59 (2012) 521-529.

20. M. Stiemer, J. Unger, B. Svendsen, H. Blum, An arbitrary Lagrangian Eulerian approach to the three-dimensional simulation of electromagnetic forming, Comput. Methods Appl. Mech. Engrg. 198 (2009) 1535-1547.

21. J. Unger, M. Stiemer, M. Schwarze, B. Svendsen, H. Blum, S. Reese, Strategies for 3D simulation of electromagnetic forming processes, J. Mater. Process. Technol. 199 (2008) 341-362.

22. S. Reese, B. Svendsen, M. Stiemer, J. Unger, M. Schwarze, H. Blum, On a new finite element technology for electromagnetic metal forming processes, Arch. Appl. Mech. 74 (11-12) (2005) 834-845.

23. F.G. Flores, A "Prism" solid element for large strain shell analysis, Comput. Methods Appl. Mech. Engrg. 253 (2013) 274-286.

24. F.G. Flores, Development of a non-linear triangular prism solid-shell element using ANS and EAS techniques, Comput. Methods Appl. Mech. Engrg. 266 (2013) 81-97.

25. D. Wang, J. S. Chen, A Hermite reproducing kernel approximation for thin-plate analysis with sub-domain stabilized conforming integration. Int. J. Numer. Meth. Eng, 74 (3) (2008) 368-390.

26. D. Wang, H. Peng, A Hermite reproducing kernel Galerkin meshfree approach for buckling analysis of thin plates. Comput. Mech. 51 (6) (2013) 1013-1029.

27. H. Feng, X.Y. Cui, G.Y. Li, A stable nodal integration method with strain gradient for static and dynamic analysis of solid mechanics, Eng. Anal. Bound. Elem. 62 (2016) 78-92.

28. K.U. Bletzinger, M. Bischoff, E. Ramm, A unified approach for shear-locking free triangular and rectangular shell finite elements, Comput. Struct. 75 (2000) $321-334$. 
29. G.R. Liu, A generalized gradient smoothing technique and the smoothed bilinear form for galerkin formulation of a wide class of computational methods, Int. J. Comput. Methods. 05 (2008) 199-236.

30. D.D. Wang, J.C. Wu, An efficient nesting sub-domain gradient smoothing integration algorithm with quadratic exactness for Galerkin meshfree methods, Comput. Methods Appl. Mech. Engrg. 298 (2016) 485-519.

31. G.R. Liu, T.T. Nguyen, K.Y. Dai, K.Y. Lam, Theoretical aspects of the smoothed finite element method (SFEM), Int. J. Numer. Methods Eng. 71 (2007) 902-930.

32. G.R. Liu, K.Y. Dai, T.T. Nguyen, A Smoothed Finite Element Method for Mechanics Problems, Comput. Mech. 39 (2006) 859-877.

33. J.S. Chen, C.T. Wu, S. Yoon, A stabilized conforming nodal integration for Galerkin mesh-free methods, Int. J. Numer. Meth. Eng. 0207 (2001) 435-466.

34. G. Wang, X.Y. Cui, H. Feng, G.Y. Li, A stable node-based smoothed finite element method for acoustic problems, Comput. Methods Appl. Mech. Engrg. 297 (2015) $348-370$.

35. H. Nguyen-Xuan, T. Rabczuk, S. Bordas, J.F. Debongnie, A smoothed finite element method for plate analysis, Comput. Methods Appl. Mech. Engrg. 197 (2008) 1184-1203.

36. Q. Li, J. Soric, T. Jarak, S.N. Atluri, A locking-free meshless local Petrov-Galerkin formulation for thick and thin plates, J. Comput. Phys. 208 (2005) $116-133$.

37. J.C. Simo, R.L. Taylor, A return mapping algorithm for plane stress elastoplasticity, Int. J. Numer. Methods Eng. 22 (1986) 649-670.

38. T.J. Hughes, J.C. Simo, Computational Inelasticity, Springer, 1998.

39. B. Lan, X.H.Ye, S.C.Song, W.J. Wu, B.H.Wang, Formulation and Programming of a Dynamic Constitutive Model at High Strain Rates in ABAQUS for the 304NG Stainless Steel. Appl. Math. M. China. 36 (2015) 167-177.

40. T. Belytschko, W. K. Liu, B. Moran, Nonlinear finite elements for continua and structures, John Wiley \& Sons Inc, 2014.

41. N. Takahashi, T. Nakata, H. Morishige, Summary of results for problem 20 (3D static force problem). Compel Int. J. Comput. Math. Electr. 14 (1995) 57-75.

42. S.H. Lee, D.N. Lee, Estimation of the magnetic pressure in tube expansion by electromagnetic forming, J. Mater. Process. Technol. 57 (1996) 311-315. 
43. Y.Y. Suzuki H, Negishi H, Free expansion of tube under magnetic pressure, J. Japanese Soc. Technol. Plast. 27 (1986) 1254-1260.

44. H.G. Noh, W.J. Song, B.S. Kang, J. Kim, Two-step electromagnetic forming process using spiral forming coils to deform sheet metal in a middle-block die, Int. J. Adv. Manuf. Technol. 76 (2015) 1691-1703. 


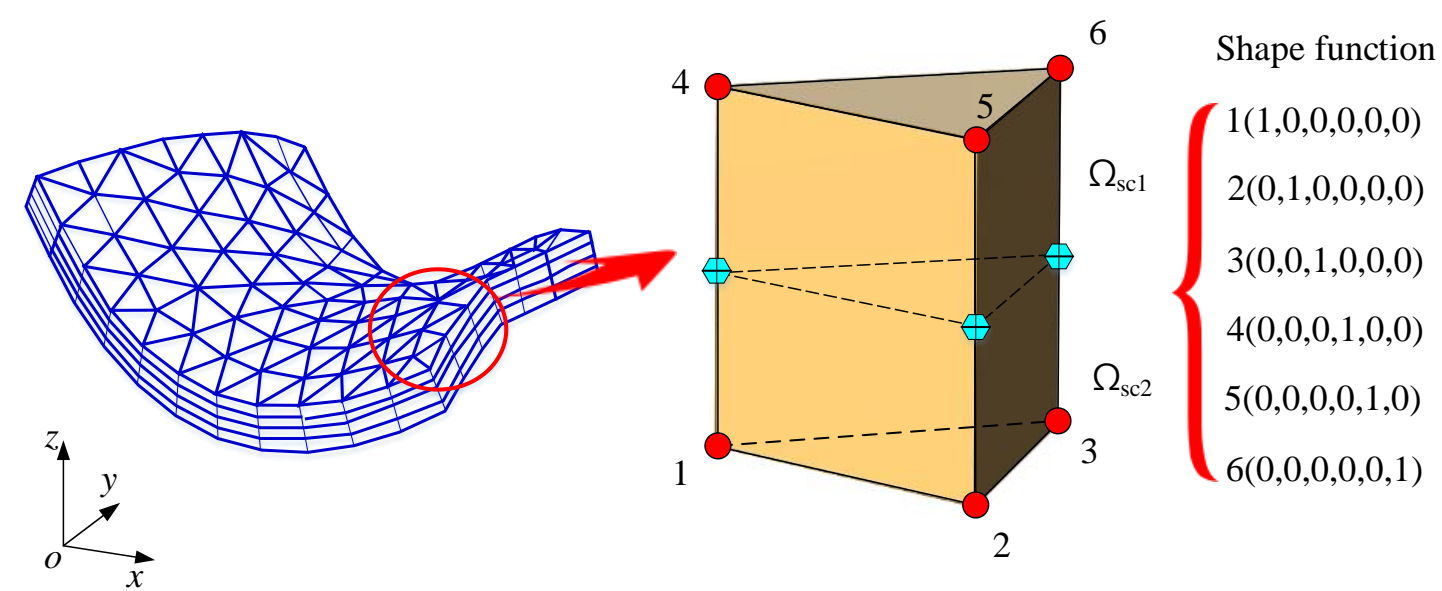

Filed node $\oplus$ Edge node

Fig. 1. The schematic of "Triprism" element

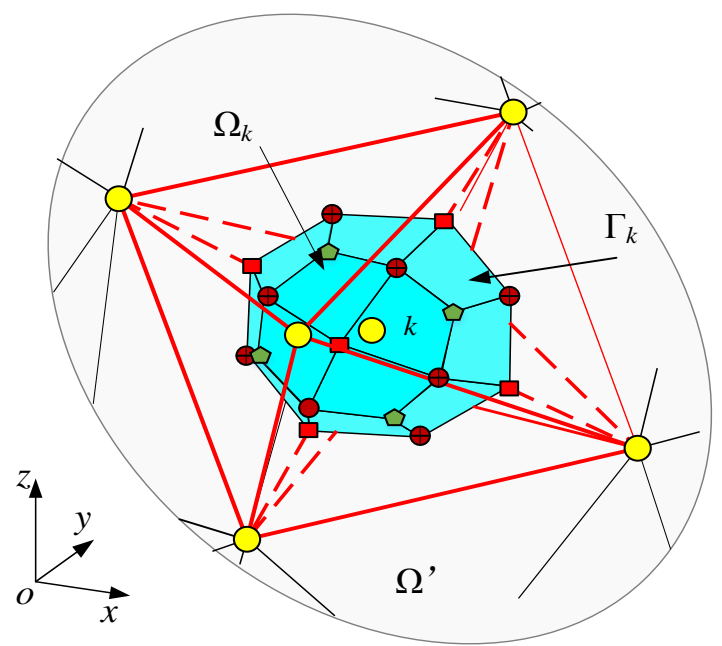

$\bigcirc \quad$ Field node

$\checkmark$ Centroid of the element

- Center of the triangle

$\square \quad$ Mid-edge-point

Fig. 2. The schematic of a node-based smoothing domain for node $\mathrm{k}$

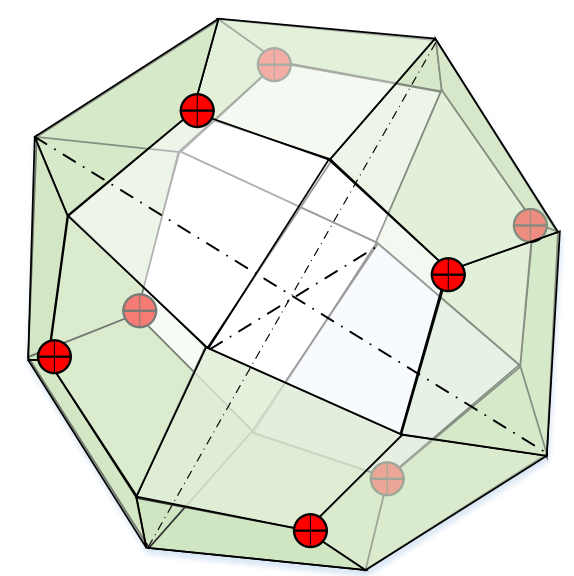

(a)

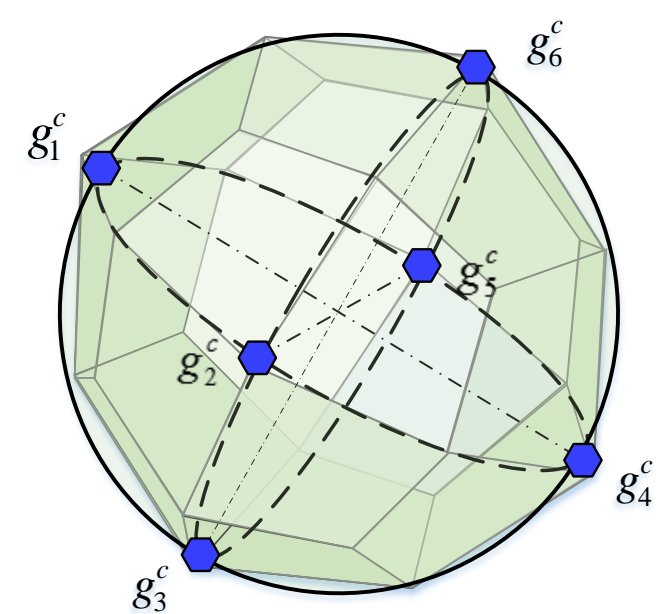

(b)

Fig. 3. The node-based smoothing domain and sphere domain. (a) Node-based smoothing domain; (b) SNIM sphere integration domain 


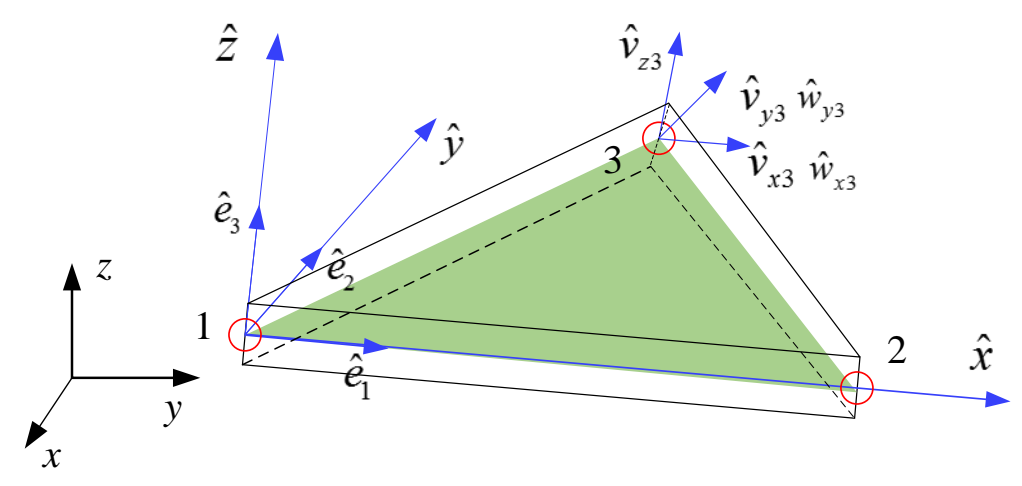

Fig. 4. A three-node triangular shell element with 5 dofs per node

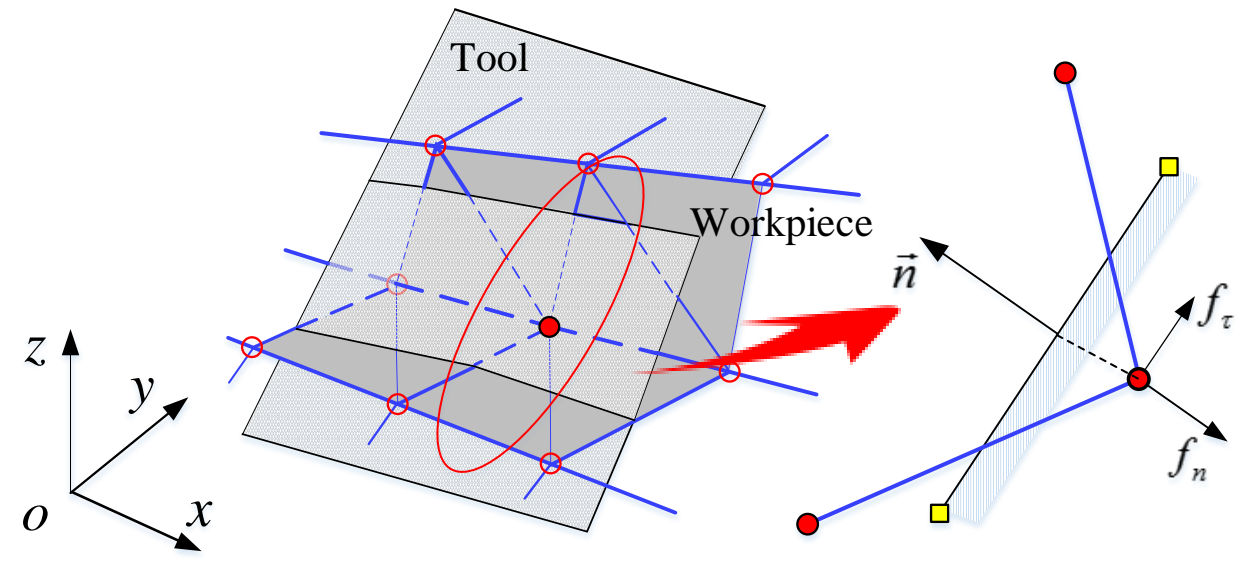

Fig. 5. Workpiece node penetrating into tool surface
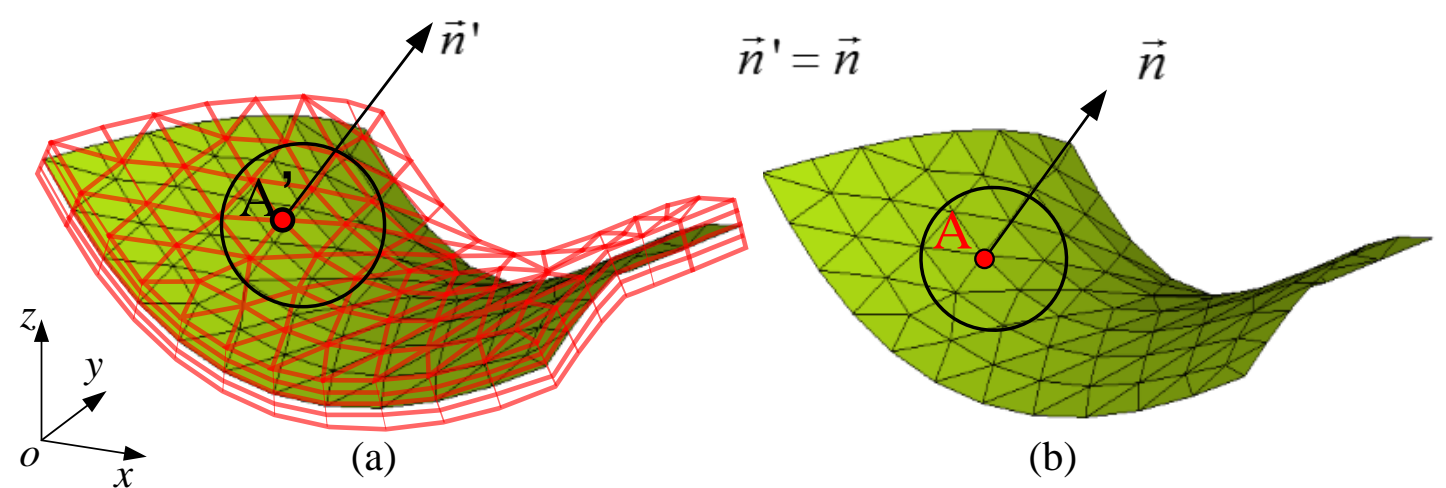

(b)

Fig. 6. Interactive mapping rule. (a). "Triprism" element in electromagnetic field; (b). Shell element in mechanical field 


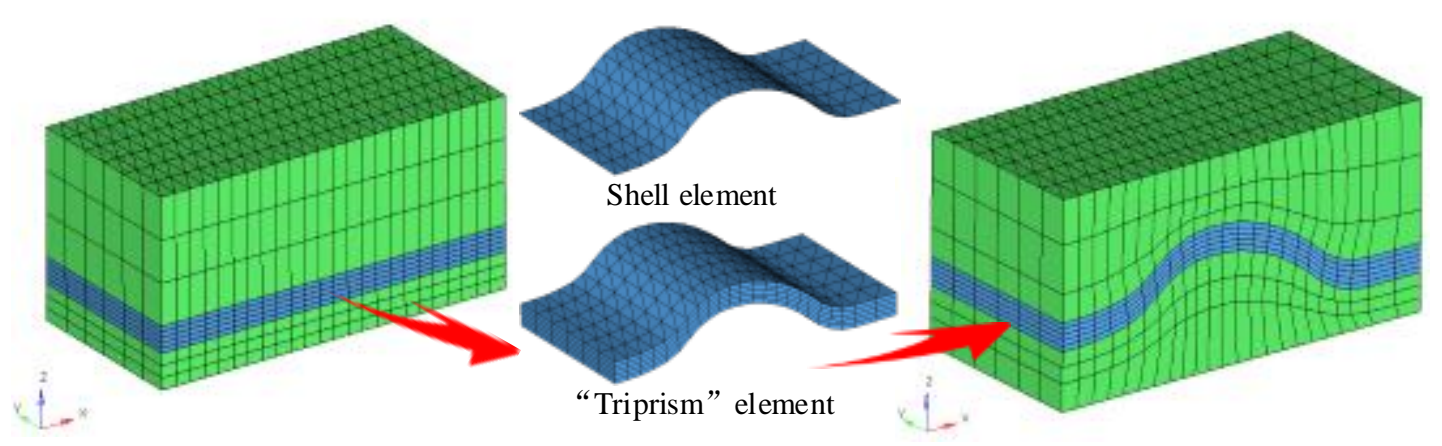

Fig. 7. The mesh before and after the deformation of sheet

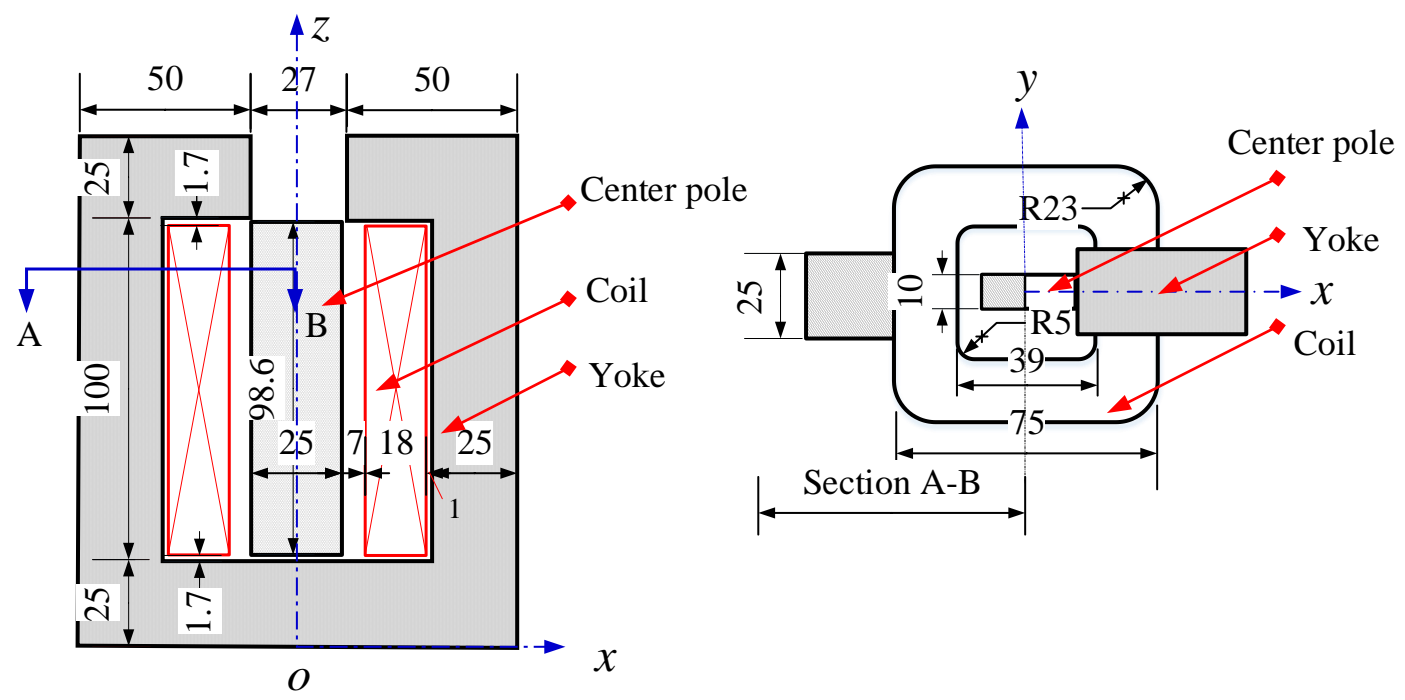

Fig. 8. 3-D model for benchmark problem 20

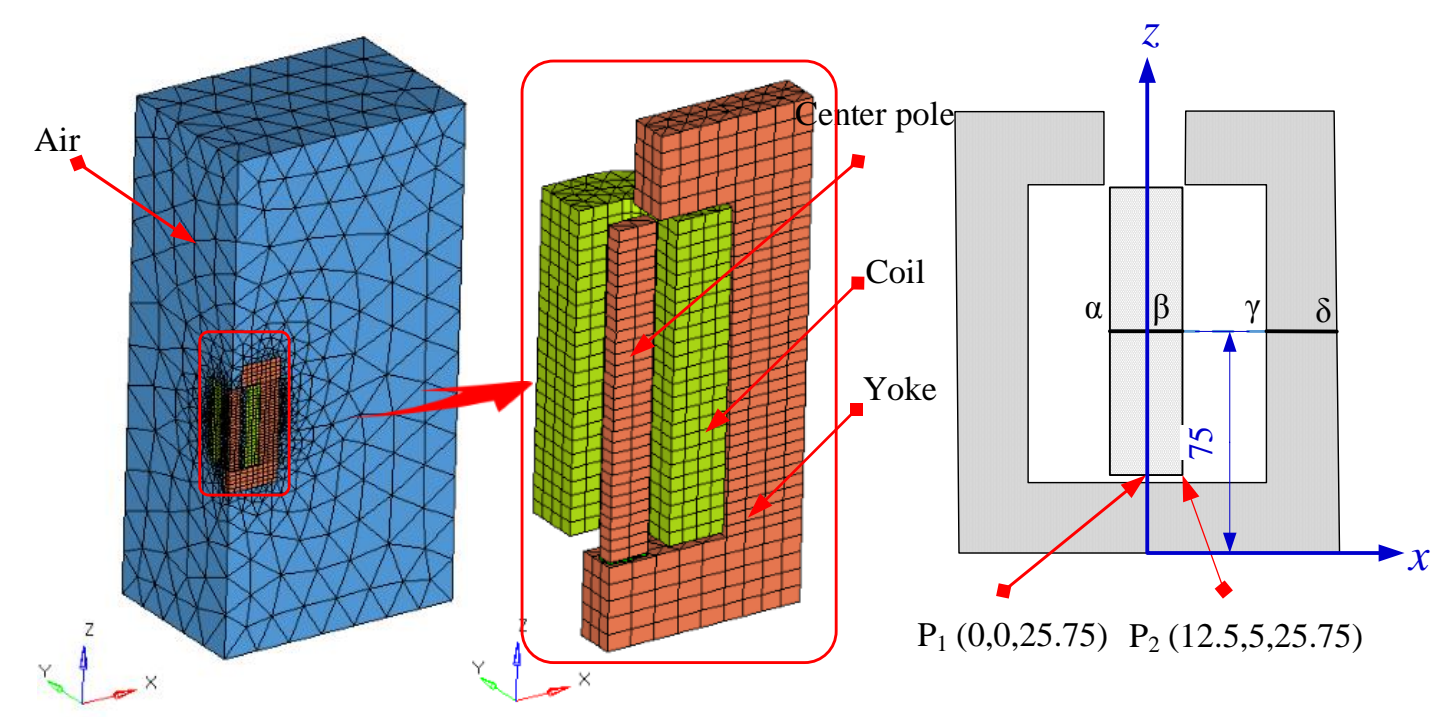

Fig. 9. Mesh model and positions at which flux densities are measured 

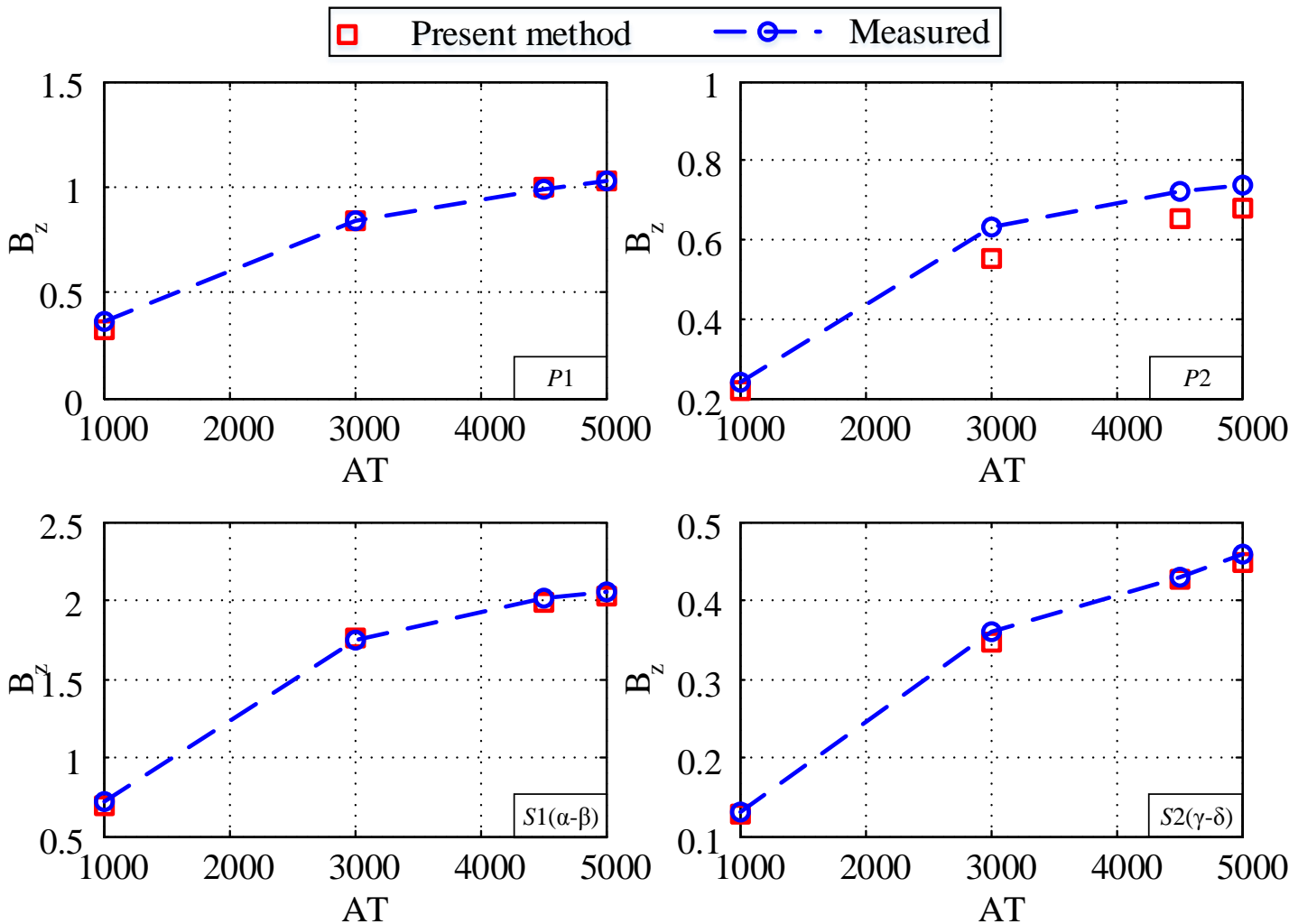

Fig. 10. Comparison of numerical results and measured ones
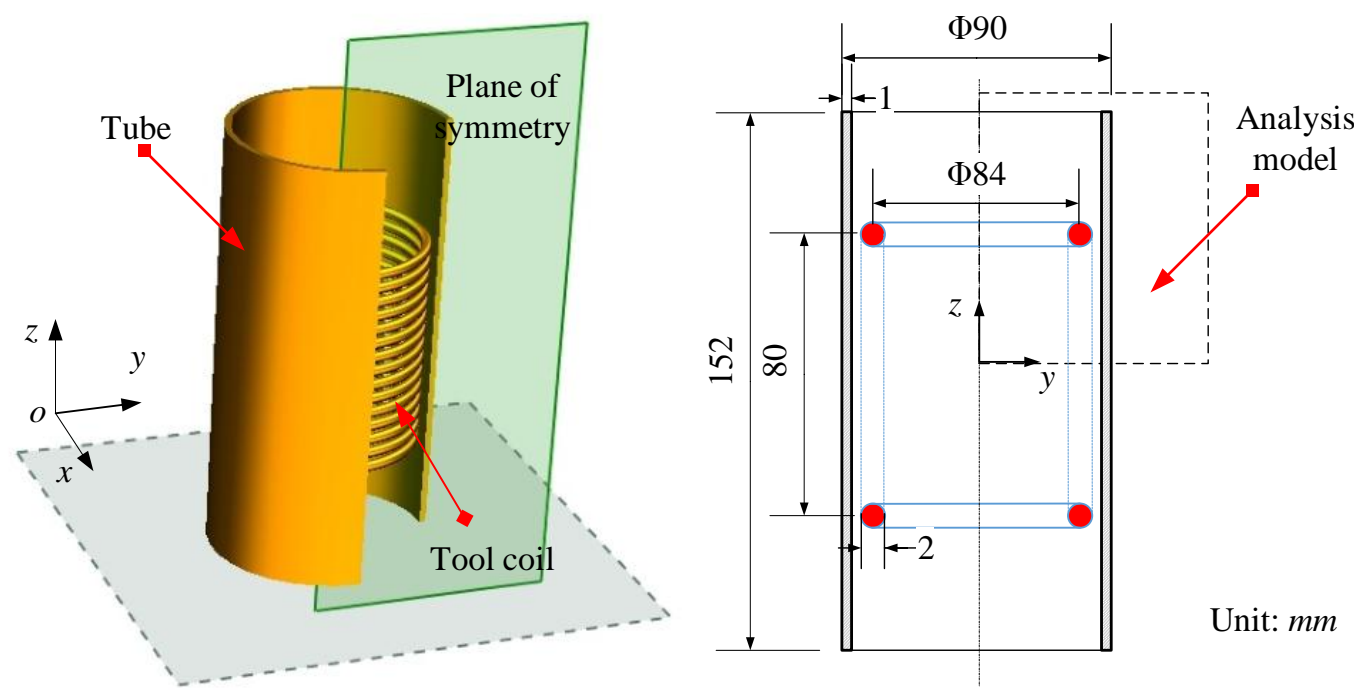

Fig. 11. Tube expansion system in electromagnetic forming 


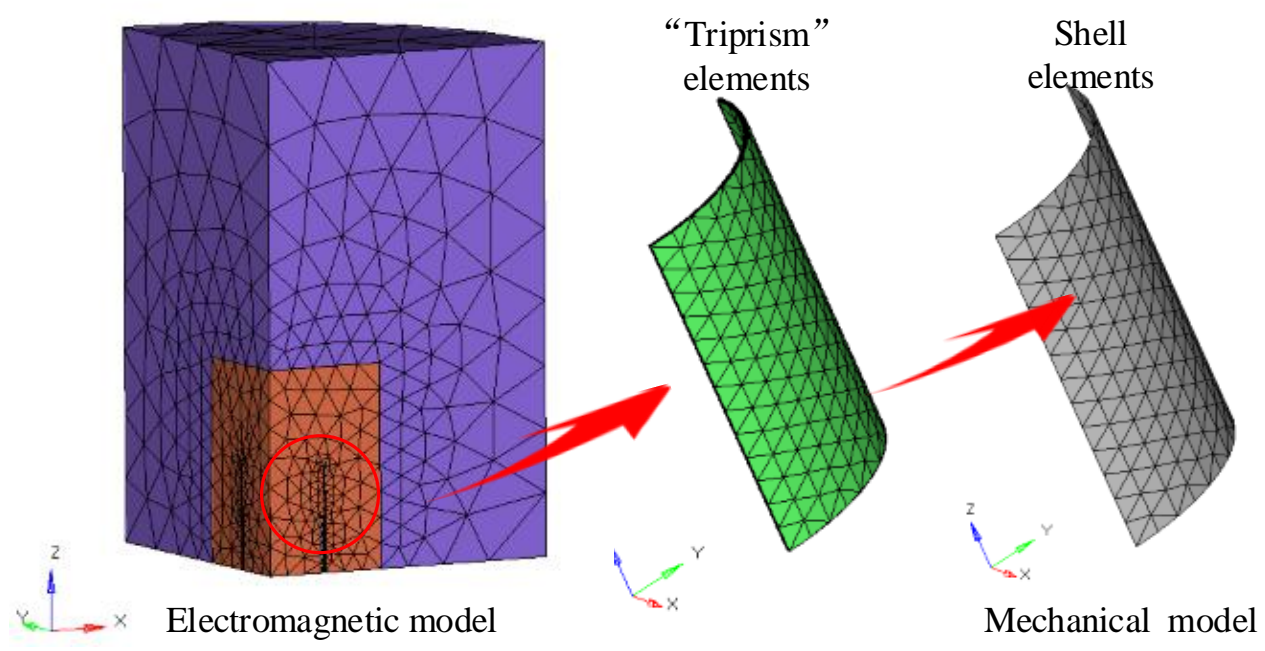

Fig. 12. Finite element mesh for tube expansion analysis

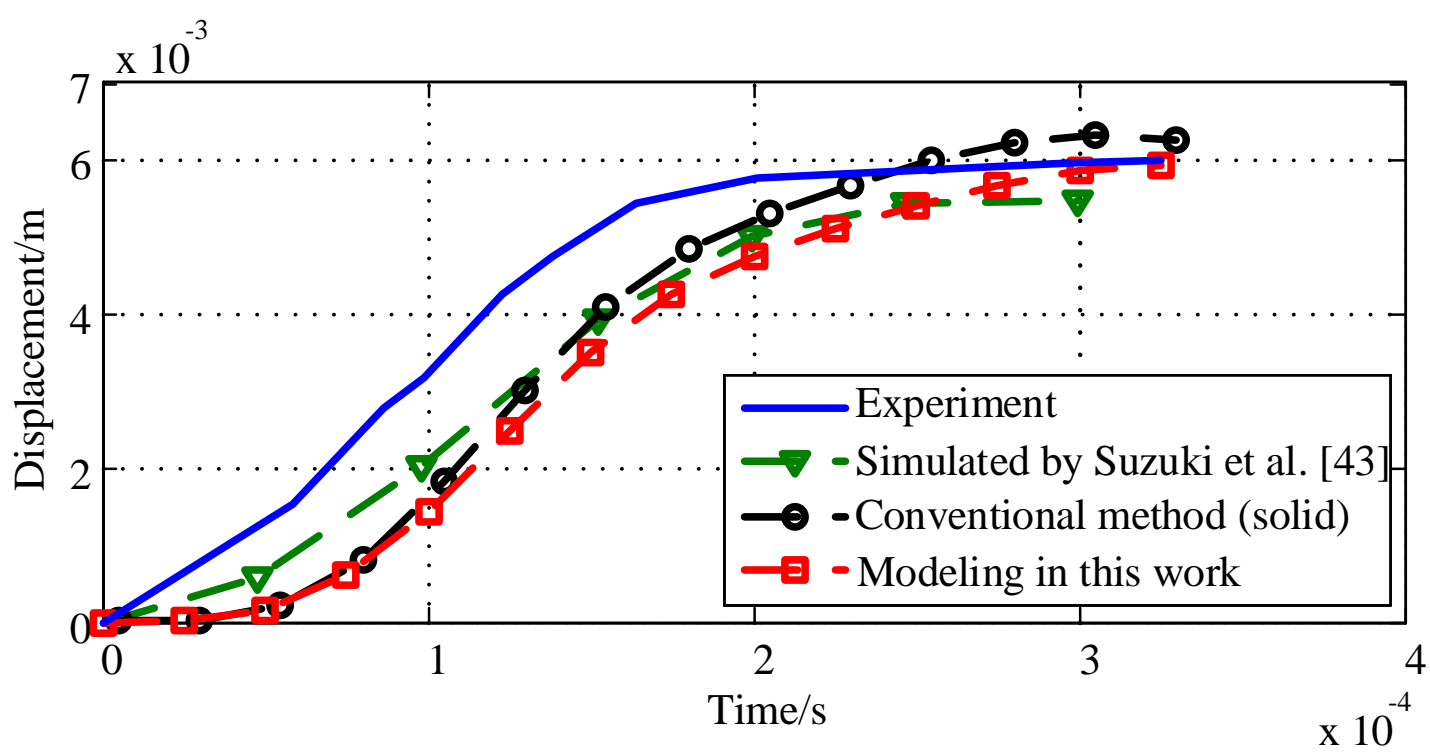

Fig. 13. Comparison of radial displacement at longitudinal center of tube 


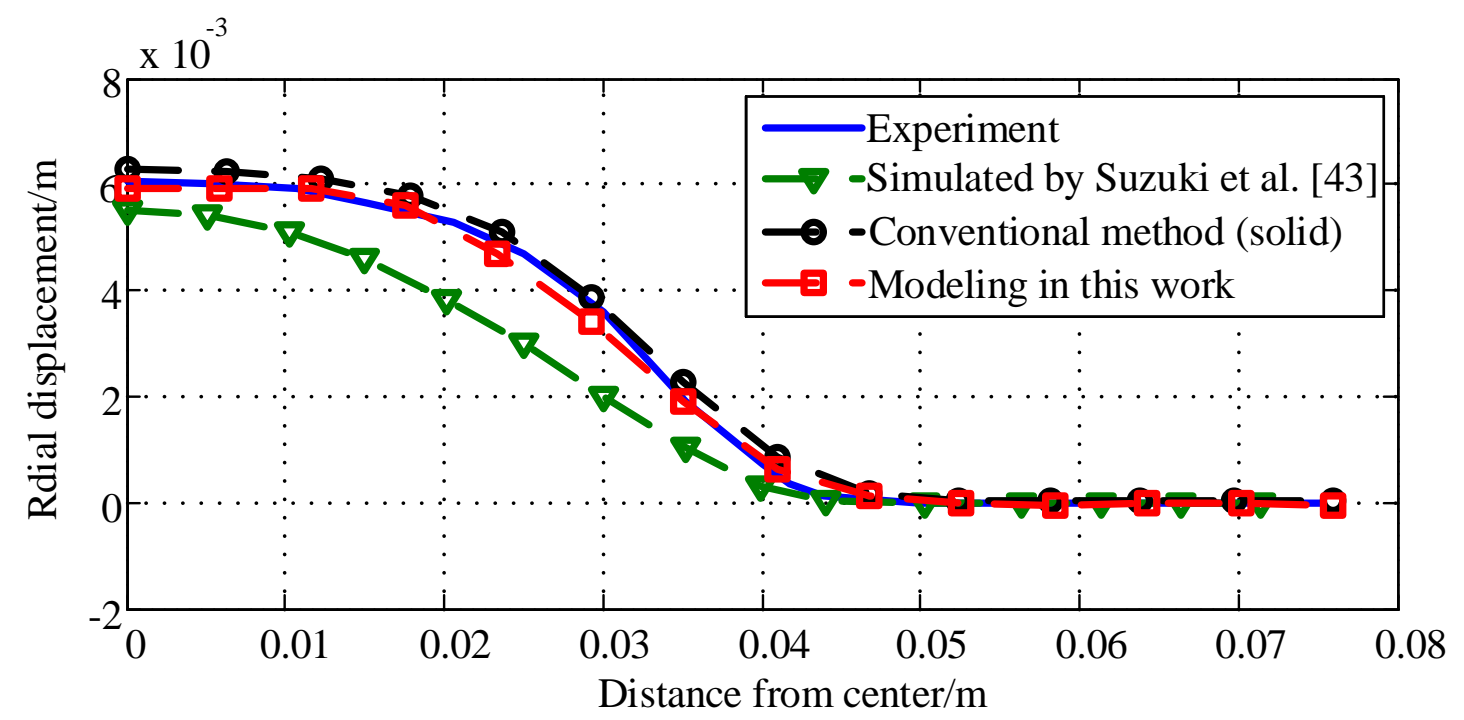

Fig. 14. Comparison of final tube shape in experiment and modeling

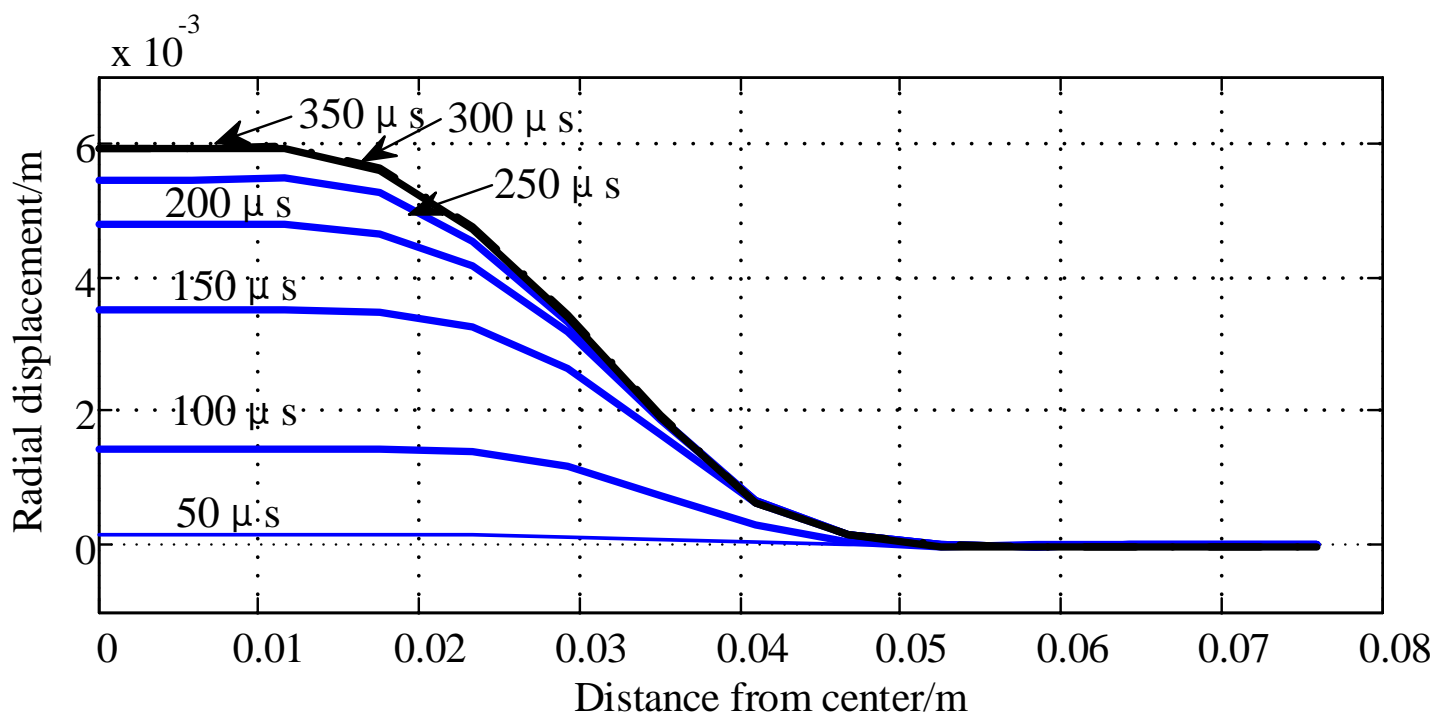

Fig. 15. Tube profile vs time 


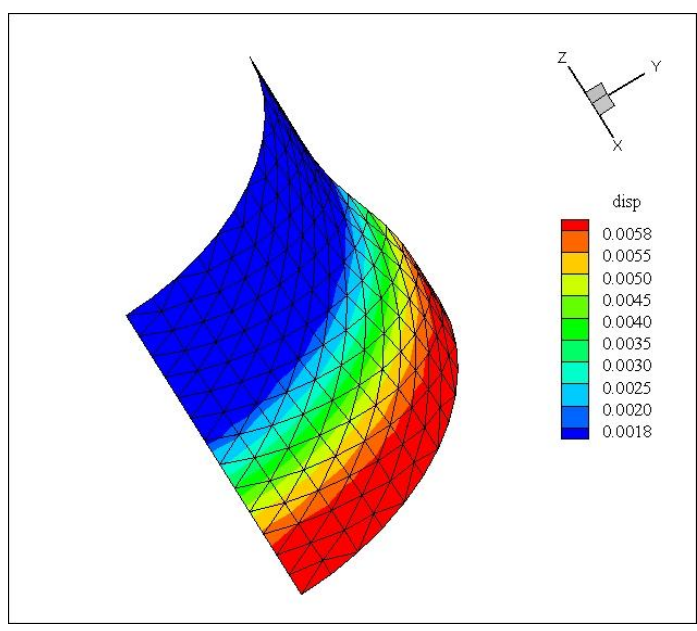

(a)

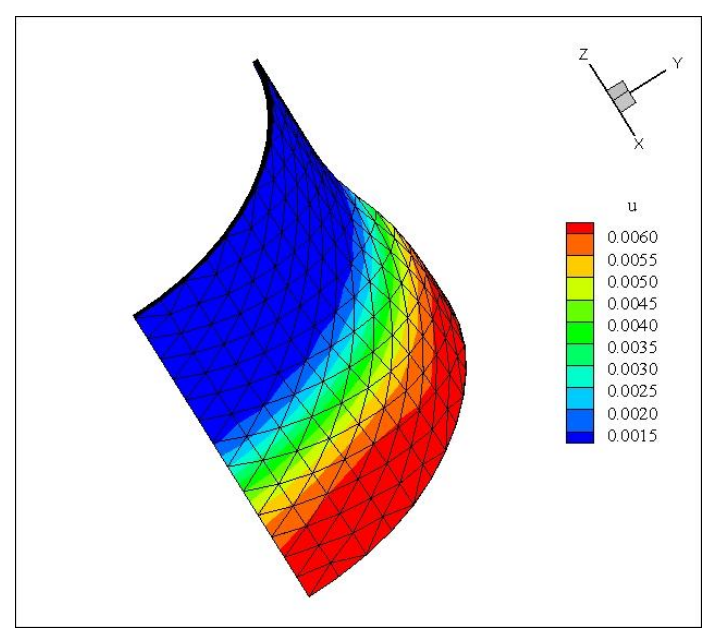

(b)

Fig. 16. Deformed tube. (a). Result by interactive mapping of "Triprism" and shell element; (b). Result by "Triprism" solid element in both electromagnetic and mechanical field

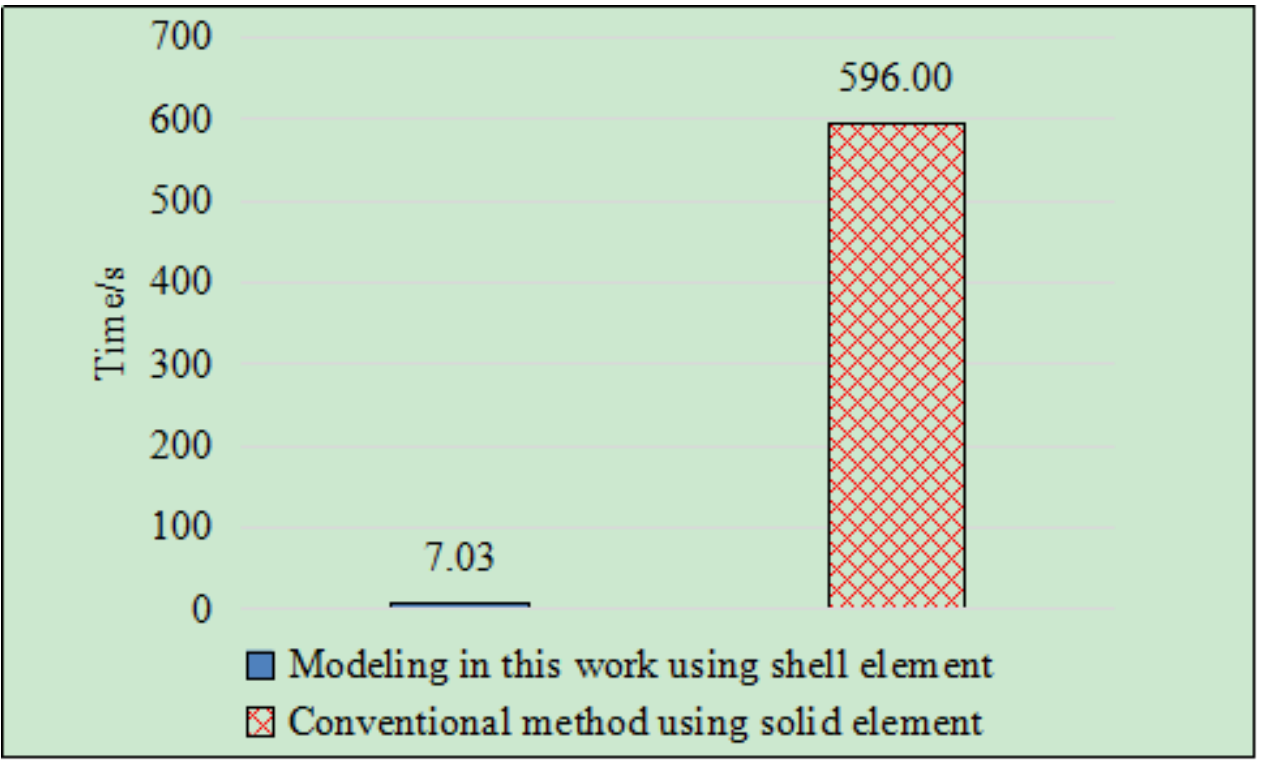

Fig. 17. Computational time of forming process in mechanical field with shell and solid element 


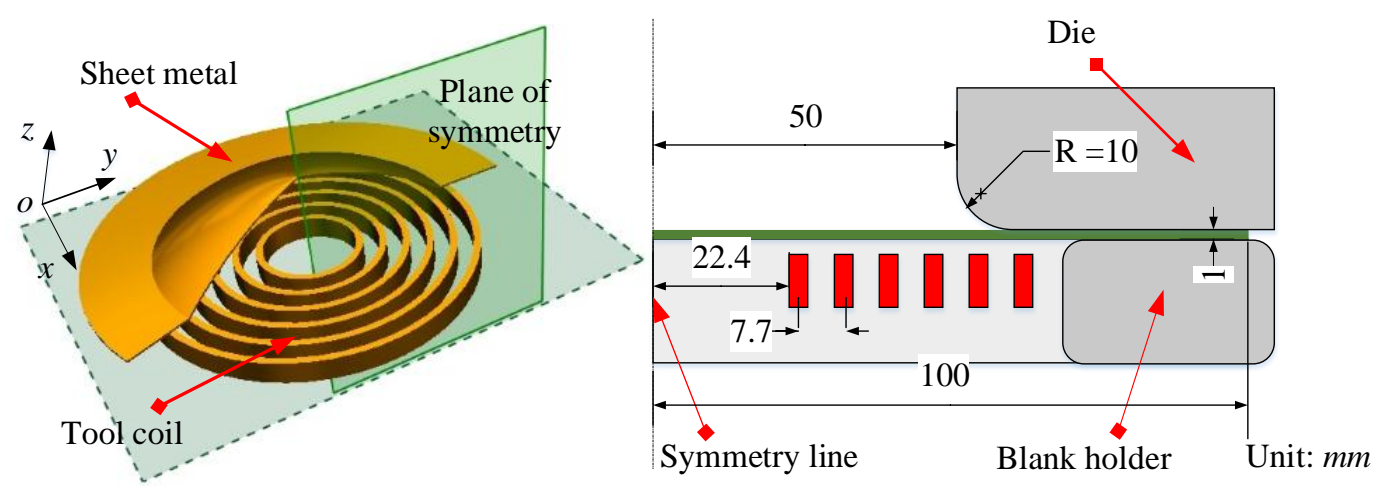

Fig. 18. System model for electromagnetic sheet metal forming

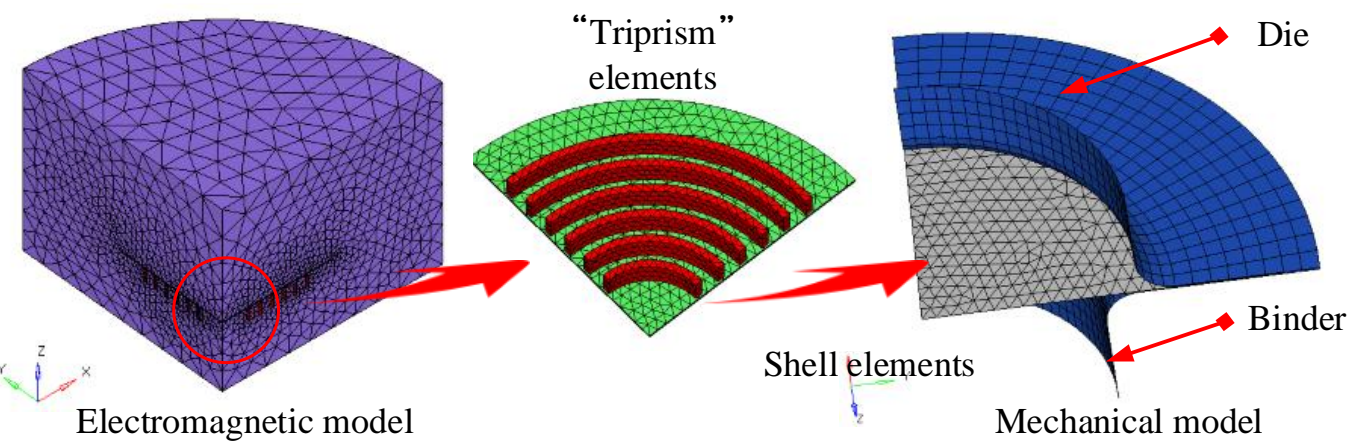

Fig. 19. Finite element mesh for sheet metal forming analysis

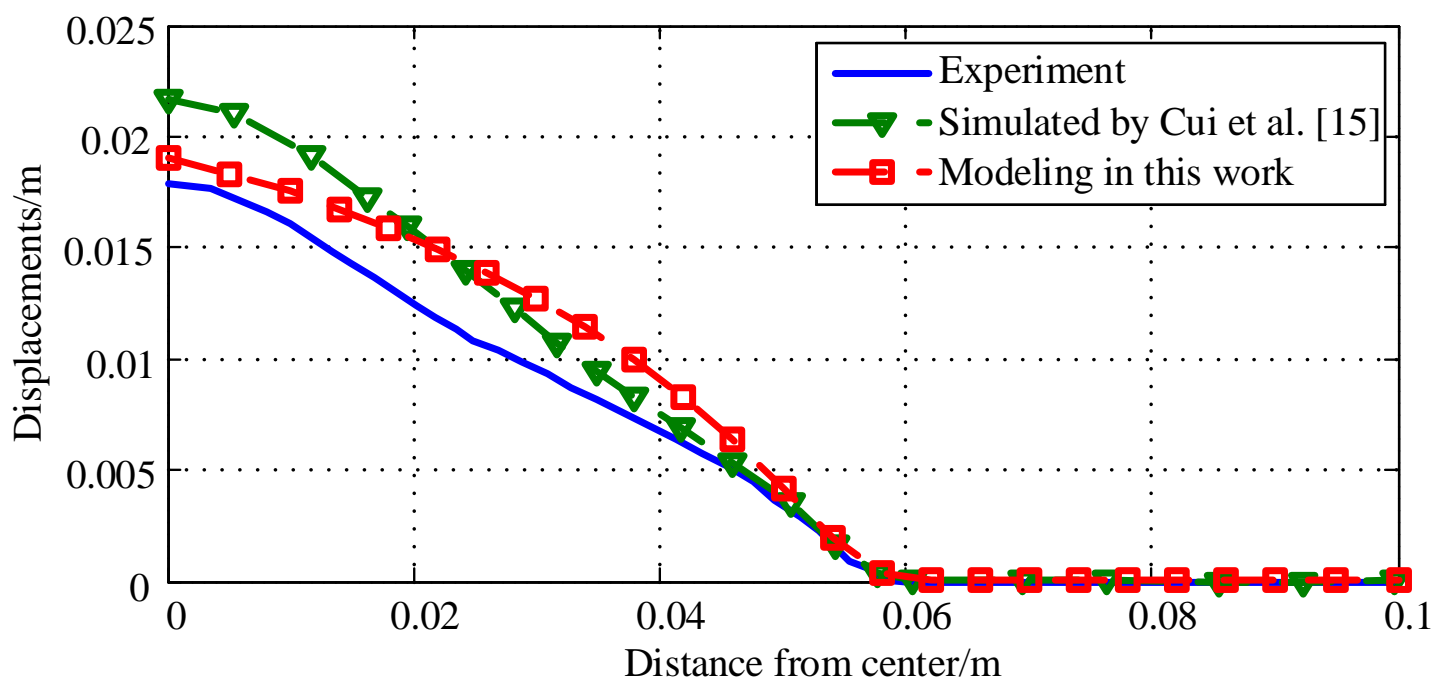

Fig. 20. Comparison of final sheet shape in experiment and modeling 


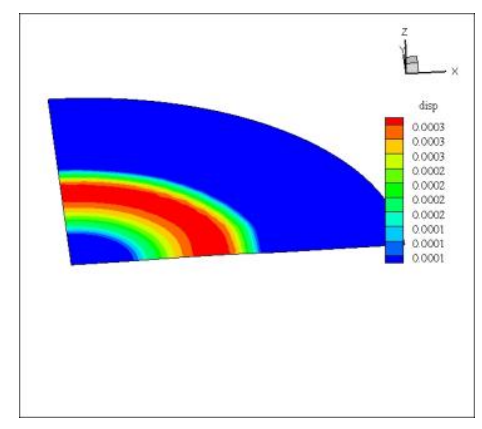

Time $=50 \mu \mathrm{s}$

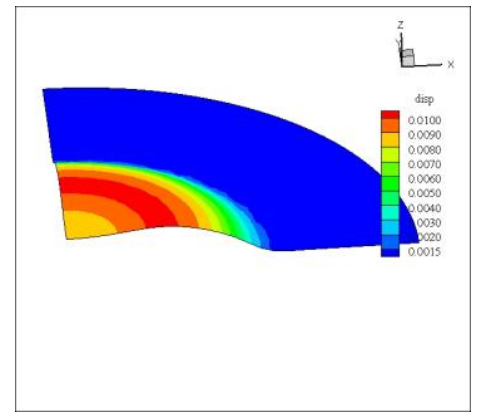

Time $=250 \mu \mathrm{s}$

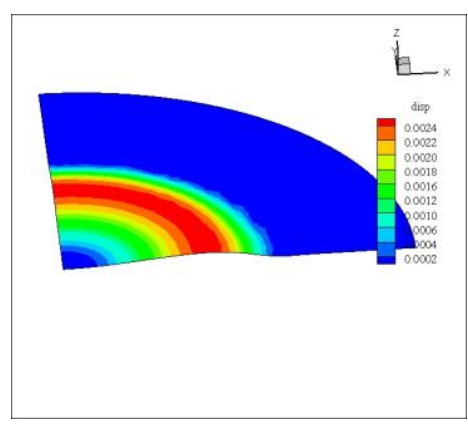

Time $=100 \mu \mathrm{s}$

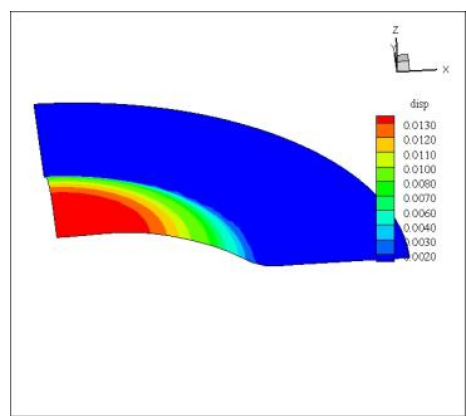

Time $=300 \mu \mathrm{s}$

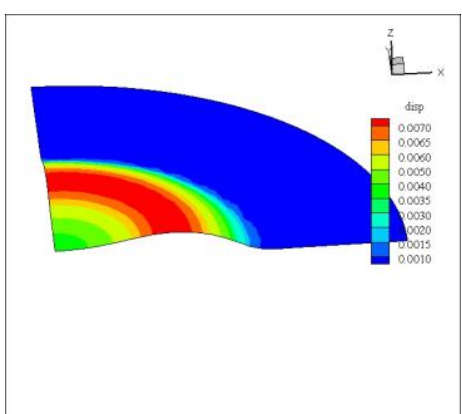

Time $=200 \mu \mathrm{s}$

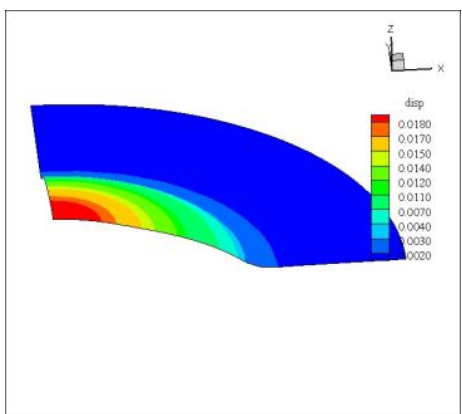

Time $=400 \mu \mathrm{s}$

Fig. 21. Sheet metal shape at different time

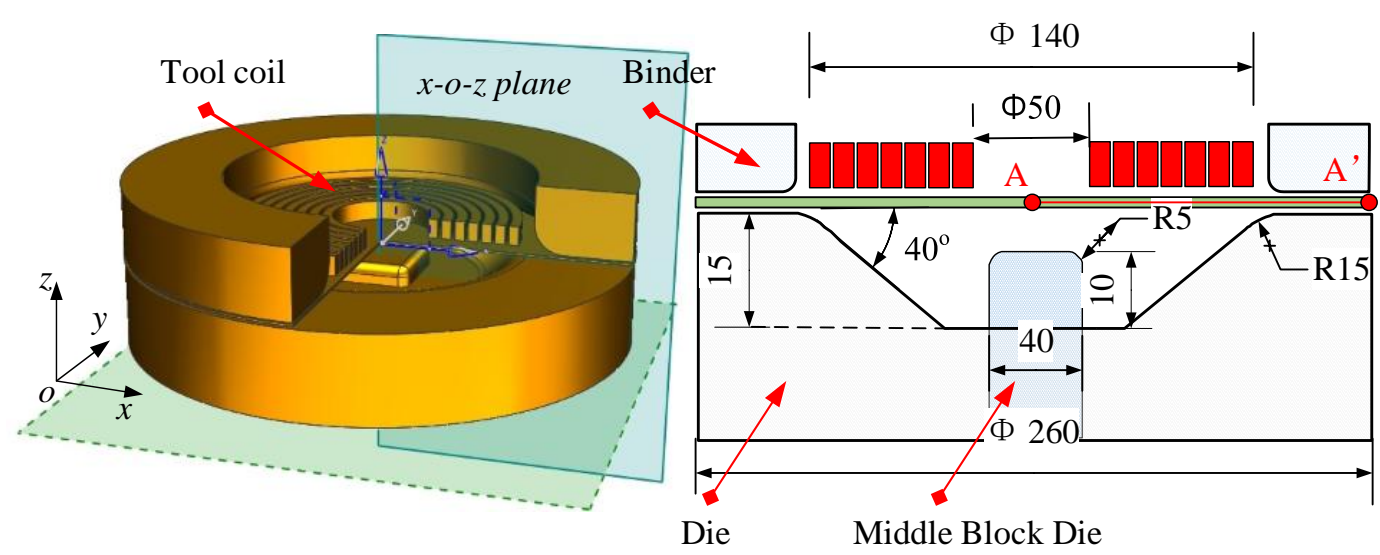

Fig. 22. System model for electromagnetic sheet forming with a middle block die 


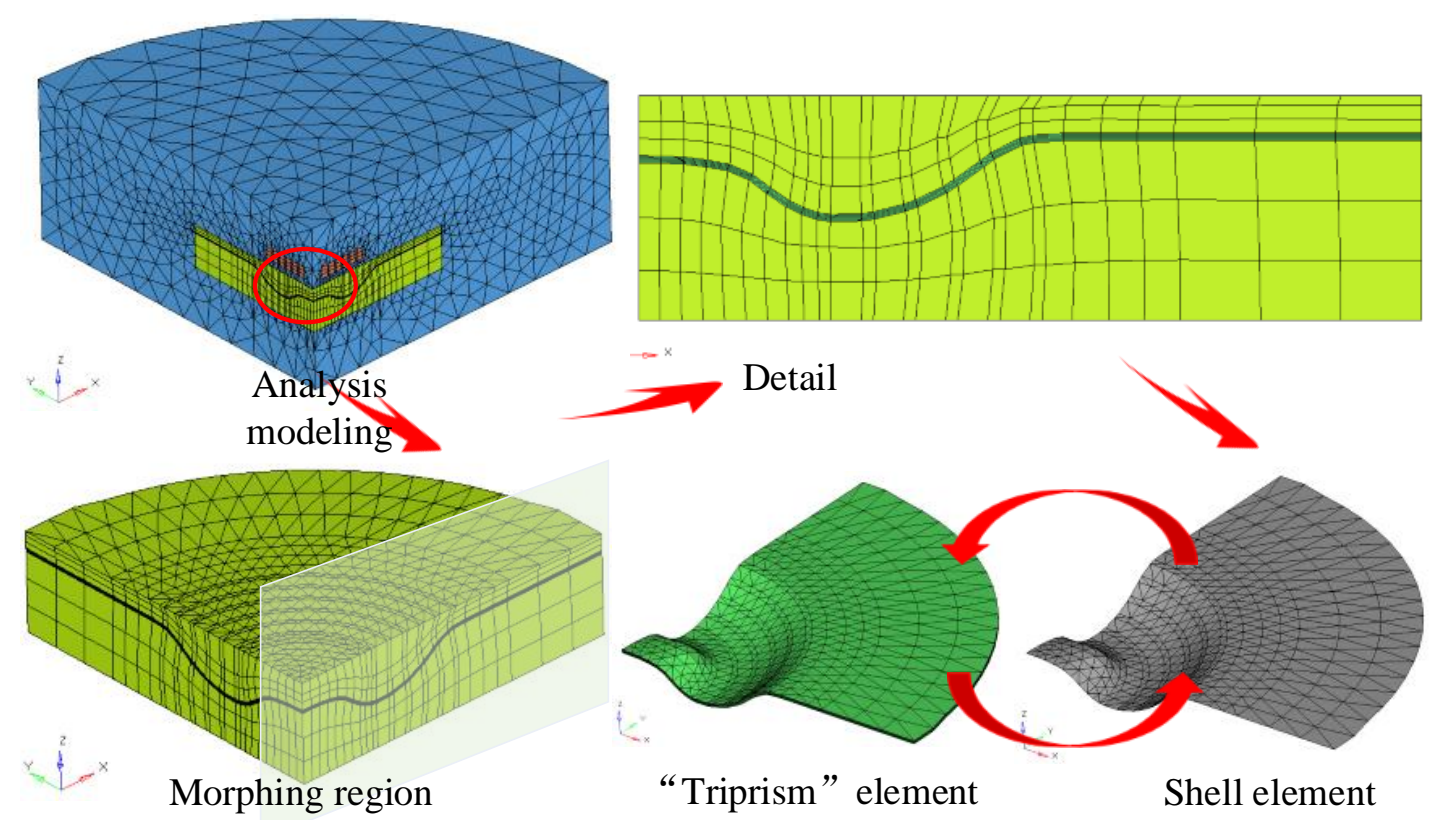

Fig. 23. Dynamic moving mesh model

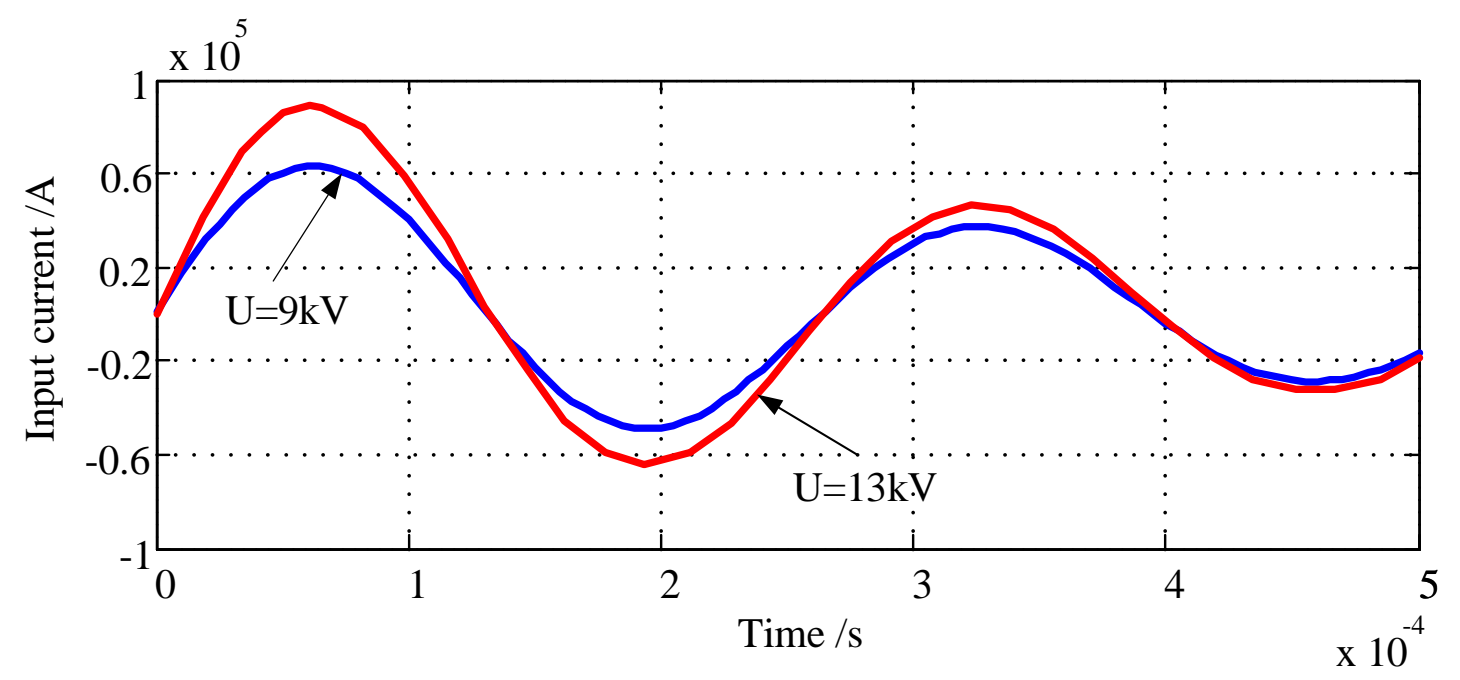

Fig. 24. Load current wave at 9 and $13 k V$ 


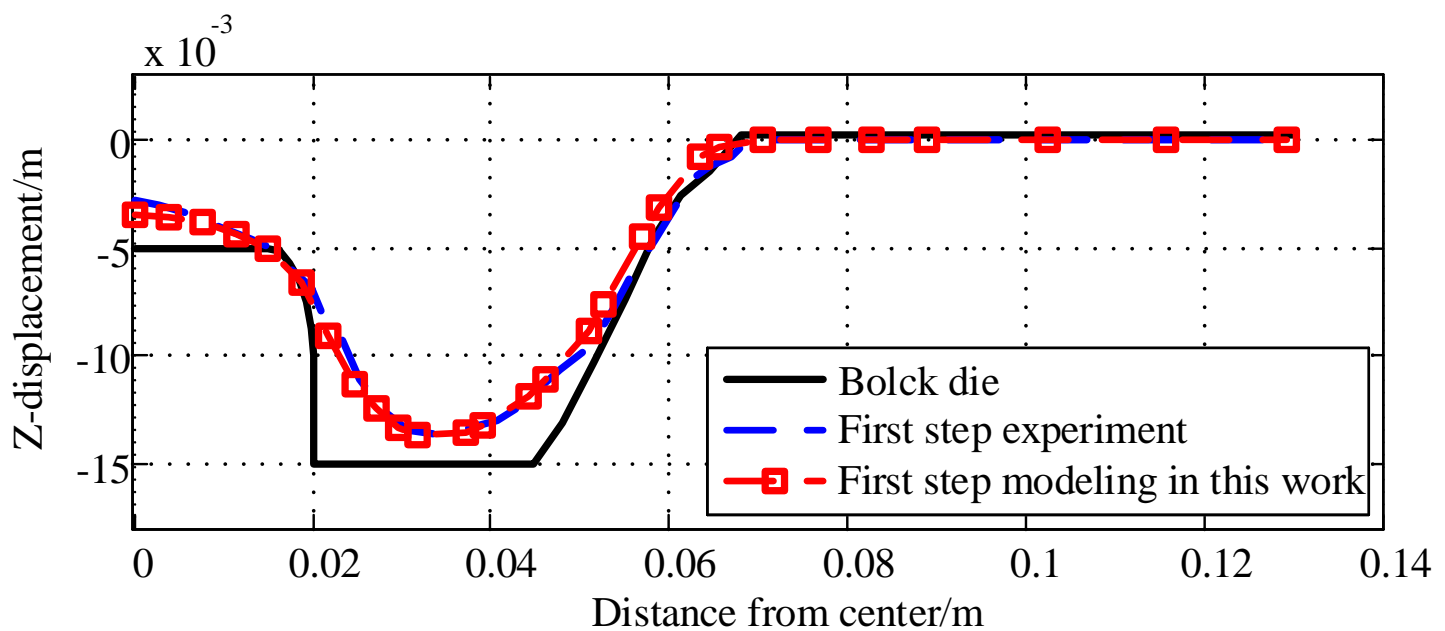

(a)

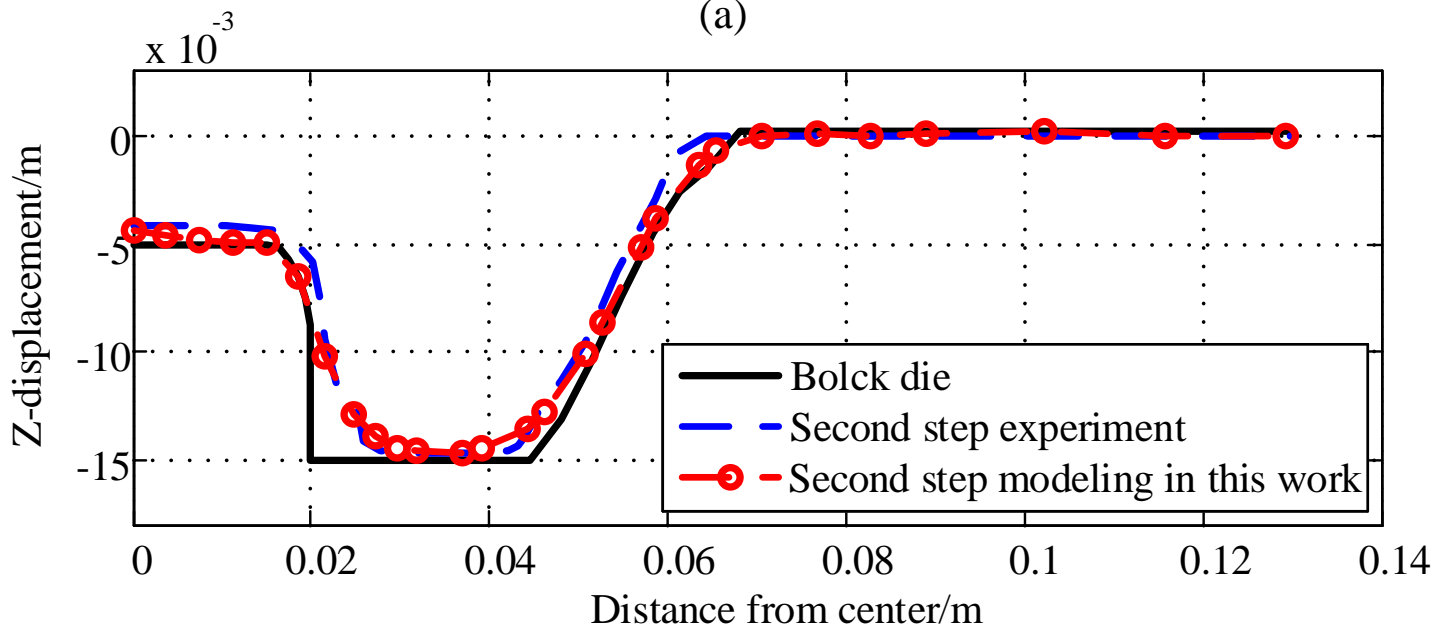

(b)

Fig. 25. Comparison of simulation results and experiment

(a). First step; (b). Second step 


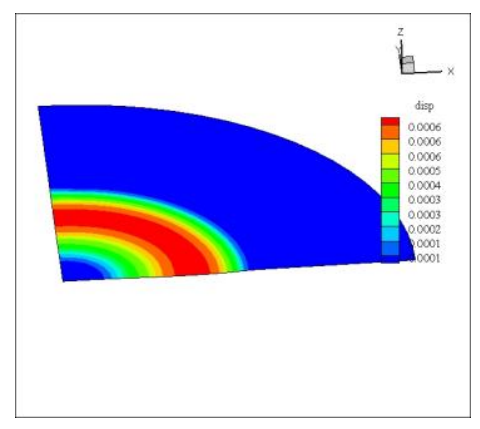

Time $=50 \mu \mathrm{s}$

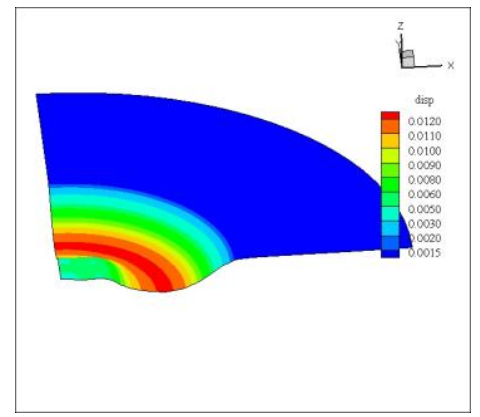

Time $=175 \mu \mathrm{s}$

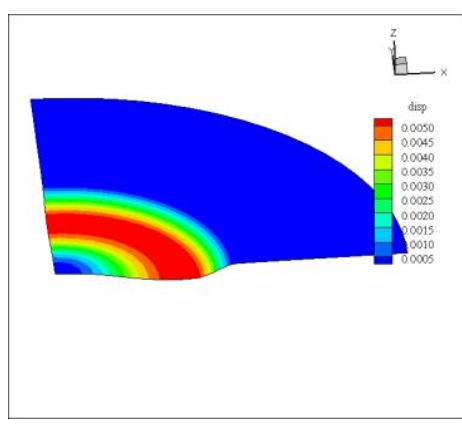

Time $=100 \mu \mathrm{s}$

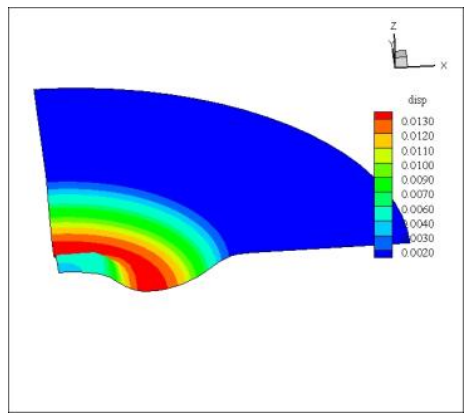

Time $=200 \mu \mathrm{s}$

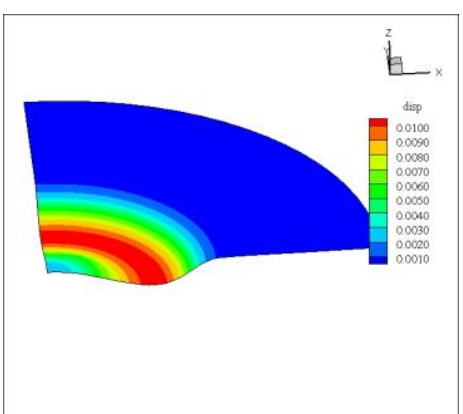

Time $=150 \mu \mathrm{s}$

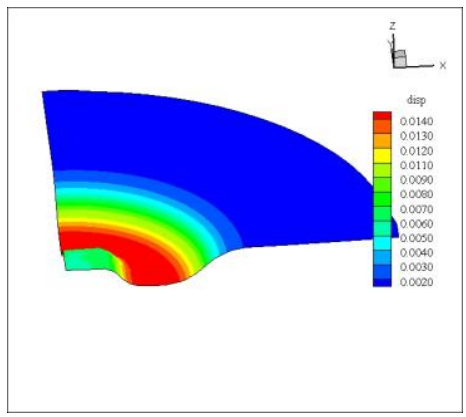

Second Step Final Shape

Fig. 26. The deformation of sheet metal at different time 
Table 1. Z-components $B z$ of flux densities at different positions

\begin{tabular}{ccccc}
\hline Position & $1000 A T$ & $3000 A T$ & $4500 A T$ & $5000 A T$ \\
\hline$P 1$ & 0.320 & 0.840 & 0.997 & 1.030 \\
$P 2$ & 0.221 & 0.555 & 0.656 & 0.679 \\
$S 1(\alpha-\beta)$ & 0.690 & 1.754 & 1.984 & 2.030 \\
$S 2(\gamma-\delta)$ & 0.129 & 0.348 & 0.428 & 0.450 \\
\hline
\end{tabular}

Table 2. Material parameters of tube expansion system

\begin{tabular}{ccc}
\hline Material & Property & Value \\
Air & Relative permeability & 1.0 \\
Coil & Relative permeability & 1.0 \\
& Density $\gamma / \mathrm{kg} \cdot \mathrm{m}^{-3}$ & 2750 \\
& Poisson's ratio $v$ & 0.3 \\
& Elastic modulus $E / G p a$ & 71.5 \\
Tube & Yield strength $\sigma_{b} / \mathrm{Mpa}$ & 8.001 \\
& & \\
& Resistivity $\rho / \Omega \cdot m$ & $2.8 \mathrm{e}-8$ \\
& Relative permeability & 1.0 \\
\hline
\end{tabular}

Table 3. Material parameters of sheet metal free bulging system

\begin{tabular}{ccc}
\hline Material & Property & Value \\
\hline Air & Relative permeability & 1.0 \\
Coil & Relative permeability & 1.0 \\
& Density $\gamma / \mathrm{kg} \cdot \mathrm{m}^{-3}$ & 2740 \\
& Poisson's ratio $v$ & 0.3 \\
Tube & Elastic modulus $E / G p a$ & 68.4 \\
$($ A3003) & Yield strength $\sigma_{b} / M p a$ & 46.8 \\
& Resistivity $\rho / \Omega \cdot m$ & $3.4 \mathrm{e}-8$ \\
& Relative permeability & 1.0 \\
\hline
\end{tabular}


Table 4. Material parameters of sheet metal forming with middle block die

\begin{tabular}{ccc}
\hline Material & Property & Value \\
Air & Relative permeability & 1.0 \\
Coil & Relative permeability & 1.0 \\
& Density $\gamma / \mathrm{kg} \cdot \mathrm{m}^{-3}$ & 2710 \\
& Poisson's ratio $v$ & 0.33 \\
Sheet metal & Elastic modulus $E / G p a$ & 68.9 \\
$($ Al-1100-O) & Resistivity $\rho / \Omega \cdot m$ & $2.90 \mathrm{e}-8$ \\
& Relative permeability & 1.0 \\
\hline
\end{tabular}

